\title{
Field dependence of the energy of a molecule in a magnetic field
}

\author{
A. Holas \\ Institute of Physical Chemistry of the Polish Academy of Sciences, 44/52 Kasprzaka, 01-224 Warsaw, Poland \\ N. H. March \\ University of Oxford, Oxford, England \\ and Department of Physics (RUCA), University of Antwerp, 2020 Antwerp, Belgium
}

(Received 10 June 1999)

\begin{abstract}
By combining the variational principle and the Hellmann-Feynman theorem, various forms of virial equations are obtained: for a many-electron system in arbitrary scalar and vector potential fields, for a neutral or charged molecule in an arbitrary vector potential field, for a molecule and an atom in a homogeneous magnetic field. These virial equations and their integrals represent exact results for the field-dependence of the groundstate and excited-state energies of the considered systems. Their validity is demonstrated not only for the exact eigenenergies, but also for a wide class of approximate energies, including the Hartree-Fock approximation and the intense-field limit of the statistical Thomas-Fermi model. [S1050-2947(99)07710-0]
\end{abstract}

PACS number(s): 31.10.+z, 33.15.-e, 95.30.Ft, 31.15.Pf

\section{INTRODUCTION}

A good deal of interest now centers on the area of atoms and molecules in intense external magnetic fields (MFs) [1-3]. Part of the motivation, at least, has come from the huge MFs that exist at the surfaces of neutron stars [4]. The problem of stability of negative molecular ions $[5,6]$ has also generated a lot of interest, which has provided additional motivation for the present study embracing molecular geometries for both neutral and charged species.

In the present investigation, a major theme is to explore the knowledge of the field-dependence of the ground-state (GS) energy of a many-electron system (in particular, a molecule) immersed in an arbitrary MF. The principal tools employed below are (a) the variational principle and (b) the Hellmann-Feynman theorem. The outline of the paper is then as follows. In Sec. II below, the Hamiltonian used throughout is defined in terms of arbitrary potential fields, as well as its expectation value in terms of low-order density matrices (DMs). This is followed in turn by a discussion of our principal tools, which leads to the virial equation (VE). Section III specializes this result to a molecule with clamped nuclei in an arbitrary MF, while Sec. IV specializes further to a homogeneous MF of arbitrary strength $B$. In that context, some attention is given to "uniform scaling," i.e., scaling all bond lengths in the molecular species by the same factor $R$. This is followed by a derivation of the GS molecular energy as a function of $R$ and $B$ in terms of the first-order DM (1DM). An alternative form for this same energy is derived in terms of the pair density (the diagonal form of 2DM). As a special, and very significant case, the field-dependence of the molecular energy is obtained at a fixed molecular geometry. The simplifications to be achieved for a single atom in a homogeneous MF are then stressed in Sec. V. To this point, all results are exact, but inevitably formal because of the appearance of fully interacting $1 \mathrm{DMs}$ and $2 \mathrm{DMs}$ in the presence of $B$. Therefore, it is of importance that for some approximate solutions of the Schrödinger equation these formally exact relations continue to be valid, as discussed at some length in Sec. VI, where, in addition to the HartreeFock method [7,8], the Thomas-Fermi statistical model [9], generalized to intense MFs [10-13], is among the approximations treated. The validity of the results for the fielddependence of the GS energy is shown to hold also for the excited-state energies. Section VII constitutes a summary of the main achievements of the present study. In Appendix A, some attention is given to questions involving gauge invariance. In connection with the magnetic-Thomas-Fermi model, it is verified in Appendix B that the known expression [12] for the approximate GS energy of an atom (as a function of the electron number $N$, the atomic number $Z$, and the MF $B$ ), satisfies the VE and the related to it extrapolating expression.

In view of the importance nowadays of the densityfunctional theory (DFT) (see, e.g., Ref. [14]), we would like to comment on DFT aspects of VEs. Since all DFT calculations of the GS molecular energy in MF bear some error due to approximate (by necessity) exchange-correlation scalar and vector potentials, the discrepancy in satisfaction of VEs and other relationships derived here may help to determine the best approximants. Our previous work [15] shows that the differential VE (which leads to the present "global" VE by means of integration) can be used to reveal properties of the field-dependent exchange-correlation potential of the DFT.

\section{ARBITRARY POTENTIAL FIELDS}

\section{A. The Hamiltonian and its expectation value in terms of density matrices}

The nonrelativistic Hamiltonian for a system of $N$ interacting electrons moving in the fields of the external scalar potential $\mathrm{v}(\mathbf{r})$, and of the vector potential $\mathbf{A}(\mathbf{r})$, which defines the MF (the magnetic flux density) via

$$
\mathbf{B}(\mathbf{r} ;[\mathbf{A}])=\boldsymbol{\nabla}(\mathbf{r}) \times \mathbf{A}(\mathbf{r}),
$$

is given by 


$$
\begin{aligned}
\hat{H}[\mathbf{v}, \mathbf{A}]= & \sum_{j=1}^{N}\left\{\frac{1}{2 m}\left(-i \hbar \boldsymbol{\nabla}\left(\mathbf{r}_{j}\right)+e \mathbf{A}\left(\mathbf{r}_{j}\right)\right)^{2}\right. \\
& \left.+\frac{g e \hbar}{2 m} \hat{\mathbf{s}}\left(\sigma_{j}\right) \cdot \mathbf{B}\left(\mathbf{r}_{j} ;[\mathbf{A}]\right)+\mathrm{v}\left(\mathbf{r}_{j}\right)\right\}+\sum_{i<j}^{N} u\left(\mathbf{r}_{i}, \mathbf{r}_{j}\right) .
\end{aligned}
$$

Here $\boldsymbol{\nabla}(\mathbf{r})=\partial / \partial \mathbf{r}, \mathbf{x}_{j} \equiv\left\{\mathbf{r}_{j}, \sigma_{j}\right\}$ denotes the space-spin coordinate of the $j$ th electron, $\{m,-e\}$ are its mass and charge, $\hat{\mathbf{s}}(\sigma)$ is the spin angular momentum operator (its $z$ component having the eigenvalues $\left.\pm \frac{1}{2}\right), g=2.0023$ is the electron Landé factor, and

$$
u\left(\mathbf{r}_{i}, \mathbf{r}_{j}\right)=\frac{e^{2}}{4 \pi \varepsilon_{0}} \frac{1}{\left|\mathbf{r}_{i}-\mathbf{r}_{j}\right|}
$$

is the Coulombic electron-electron interaction potential. To simplify the notation, we suppress the dependence on fixed $N$ in writing the arguments of $\hat{H}$, and, next, of all related quantities. The SI convention for units and electromagnetic relations is used.

The expectation value of $\hat{H}$ with respect to $\Psi\left(\mathbf{x}_{1}, \mathbf{x}_{2}, \ldots, \mathbf{x}_{N}\right)-$ an antisymmetric, normalized wave function $\langle\Psi \mid \Psi\rangle=1$ - can be written in terms of low-order DMs as

$$
\begin{aligned}
\langle\Psi|\hat{H}[\mathrm{~V}, \mathbf{A}]| \Psi\rangle= & \widetilde{T}_{0}\left[\rho_{1}\right]+\widetilde{T}_{1}\left[\rho_{1}, \mathbf{A}\right]+\widetilde{T}_{2}\left[n, \mathbf{A}^{2}\right] \\
& +\widetilde{T}_{1},\left[\gamma_{1}, \mathbf{A}\right]+\widetilde{V}[n, \mathrm{~V}]+\widetilde{E}_{e e}\left[n_{2}, u\right]
\end{aligned}
$$

where four kinetic-energy terms $\widetilde{T}_{0}, \widetilde{T}_{1}, \widetilde{T}_{2}$, and $\widetilde{T}_{1}$, due to the Hamiltonian terms - free of $\mathbf{A}$, linear in $\mathbf{A}$, quadratic in $\mathbf{A}$, and spin-MF interaction, respectively — are functionals of the 1DM:

$$
\begin{aligned}
& \widetilde{T}_{0}\left[\rho_{1}\right]=\int d^{3} r t\left(\mathbf{r} ;\left[\rho_{1}\right]\right)>0, \\
& \widetilde{T}_{1}\left[\rho_{1}, \mathbf{A}\right]=\int d^{3} r e \mathbf{j}_{\mathrm{p}}\left(\mathbf{r} ;\left[\rho_{1}\right]\right) \cdot \mathbf{A}(\mathbf{r}), \\
& \widetilde{T}_{2}\left[n, \mathbf{A}^{2}\right]=\int d^{3} r n(\mathbf{r}) \frac{e^{2}}{2 m} \mathbf{A}^{2}(\mathbf{r})>0, \\
& \widetilde{T}_{1},\left[\gamma_{1}, \mathbf{A}\right]=-\int d^{3} r \mathbf{m}\left(\mathbf{r} ;\left[\gamma_{1}\right]\right) \cdot \mathbf{B}(\mathbf{r},[\mathbf{A}]) \\
& =-\int d^{3} r \mathbf{A}(\mathbf{r}) \cdot\left\{\boldsymbol{\nabla}(\mathbf{r}) \times \mathbf{m}\left(\mathbf{r} ;\left[\gamma_{1}\right]\right)\right\} .
\end{aligned}
$$

The terms $\widetilde{V}$ and $\widetilde{E}_{\text {ee }}$ represent the electron-(external-scalarpotential) interaction energy and the electron-electron interaction energy:

$$
\widetilde{V}[n, \mathrm{v}]=\int d^{3} r n(\mathbf{r}) \mathrm{v}(\mathbf{r})
$$

$$
\widetilde{E}_{\mathrm{ee}}\left[n_{2}, u\right]=\int d^{3} r_{1} d^{3} r_{2} n_{2}\left(\mathbf{r}_{1}, \mathbf{r}_{2}\right) u\left(\mathbf{r}_{1}, \mathbf{r}_{2}\right)>0
$$

Vanishing of the DMs and their derivatives at infinite distances has been used.

The functionals of the one-body DMs, involved in $\widetilde{T}_{0}, \widetilde{T}_{1}$, and $\widetilde{T}_{1}$, are defined

$$
t\left(\mathbf{r} ;\left[\rho_{1}\right]\right)=\left.\frac{\hbar^{2}}{2 m} \boldsymbol{\nabla}\left(\mathbf{r}^{\prime}\right) \cdot \boldsymbol{\nabla}\left(\mathbf{r}^{\prime \prime}\right) \rho_{1}\left(\mathbf{r}+\mathbf{r}^{\prime} ; \mathbf{r}+\mathbf{r}^{\prime \prime}\right)\right|_{\mathbf{r}^{\prime}=\mathbf{r}^{\prime \prime}=\mathbf{0}} \geqslant 0
$$

for the conventional kinetic energy density,

$$
\begin{aligned}
\mathbf{j}_{\mathrm{p}}\left(\mathbf{r} ;\left[\rho_{1}\right]\right) & =-\left.\frac{i \hbar}{2 m}\left\{\boldsymbol{\nabla}\left(\mathbf{r}^{\prime}\right)-\boldsymbol{\nabla}\left(\mathbf{r}^{\prime \prime}\right)\right\} \rho_{1}\left(\mathbf{r}+\mathbf{r}^{\prime} ; \mathbf{r}+\mathbf{r}^{\prime \prime}\right)\right|_{\mathbf{r}^{\prime}=\mathbf{r}^{\prime \prime}=\mathbf{0}} \\
& =\left.\frac{\hbar}{m} \nabla\left(\mathbf{r}^{\prime}\right) \operatorname{Im} \rho_{1}\left(\mathbf{r}+\mathbf{r}^{\prime} ; \mathbf{r}\right)\right|_{\mathbf{r}^{\prime}=\mathbf{0}}
\end{aligned}
$$

for the paramagnetic-current density vector, and

$$
\mathbf{m}\left(\mathbf{r} ;\left[\gamma_{1}\right]\right)=-\left.\frac{g e \hbar}{2 m} \sum_{\sigma} \hat{\mathbf{S}}(\sigma) \gamma_{1}\left(\mathbf{r}, \sigma ; \mathbf{r}, \sigma^{\prime}\right)\right|_{\sigma^{\prime}=\sigma}
$$

for the magnetization density vector. The DMs are defined as usual (see, e.g., Refs. [14] and [15]):

$$
\begin{gathered}
\gamma_{2}\left(\mathbf{x}_{1}, \mathbf{x}_{2} ; \mathbf{x}_{1}^{\prime}, \mathbf{x}_{2}^{\prime}\right) \\
=\frac{(N-1) N}{2} \int d \mathbf{x}_{3} \cdots d \mathbf{x}_{N} \Psi\left(\mathbf{x}_{1}, \mathbf{x}_{2}, \mathbf{x}_{3}, \ldots, \mathbf{x}_{N}\right) \\
\times \Psi^{*}\left(\mathbf{x}_{1}^{\prime}, \mathbf{x}_{2}^{\prime}, \mathbf{x}_{3}, \ldots, \mathbf{x}_{N}\right) \\
n_{2}\left(\mathbf{r}_{1}, \mathbf{r}_{2}\right)=\sum_{\sigma_{1}, \sigma_{2}} \gamma_{2}\left(\mathbf{r}_{1}, \sigma_{1}, \mathbf{r}_{2}, \sigma_{2} ; \mathbf{r}_{1}, \sigma_{1}, \mathbf{r}_{2}, \sigma_{2}\right) \geqslant 0 \\
\gamma_{1}\left(\mathbf{x}_{1} ; \mathbf{x}_{1}^{\prime}\right)=\frac{2}{N-1} \int d \mathbf{x}_{2} \gamma_{2}\left(\mathbf{x}_{1}, \mathbf{x}_{2} ; \mathbf{x}_{1}^{\prime}, \mathbf{x}_{2}\right) \\
\rho_{1}\left(\mathbf{r}_{1} ; \mathbf{r}_{1}^{\prime}\right)=\sum_{\sigma_{1}} \gamma_{1}\left(\mathbf{r}_{1}, \sigma_{1} ; \mathbf{r}_{1}^{\prime}, \sigma_{1}\right) \\
n\left(\mathbf{r}_{1}\right)=\rho_{1}\left(\mathbf{r}_{1} ; \mathbf{r}_{1}\right) \geqslant 0
\end{gathered}
$$

the last being the electron-number density.

\section{B. Variational principle}

When $\Psi=\Psi\left(\mathbf{x}_{1}, \mathbf{x}_{2}, \ldots, \mathbf{x}_{N} ;[\mathbf{v}, \mathbf{A}]\right)$ is the GS wave function of the Hamiltonian $\hat{H}$, Eq. (2.2),

$$
\hat{H}[\mathrm{v}, \mathbf{A}] \Psi[\mathrm{v}, \mathbf{A}]=E[\mathrm{v}, \mathbf{A}] \Psi[\mathrm{v}, \mathbf{A}]
$$

then Eq. (2.4) for $\langle\Psi|\hat{H}| \Psi\rangle$, with all DMs being functionals of external fields $\left\{\right.$ e.g., $\rho_{1}=\rho_{1}[\mathrm{v}, \mathbf{A}]$ via Eqs. (2.14) $\left.-(2.18)\right\}$, represents an expression for the GS energy of the system: 


$$
\begin{aligned}
E[\mathrm{v}, \mathbf{A}]= & T_{0}[\mathrm{v}, \mathbf{A}]+T_{1}[\mathrm{v}, \mathbf{A}]+T_{2}[\mathrm{v}, \mathbf{A}]+T_{1},[\mathrm{v}, \mathbf{A}] \\
& +V[\mathbf{v}, \mathbf{A}]+E_{\text {ee }}[\mathbf{v}, \mathbf{A}],
\end{aligned}
$$

where $T_{1}[\mathbf{V}, \mathbf{A}]=\widetilde{T}_{1}\left[\rho_{1}[\mathrm{~V}, \mathbf{A}], \mathbf{A}\right]$ and similarly for other terms.

On the way to obtain the VE let us define the following scaled function:

$$
\begin{aligned}
& \Psi_{\xi}\left(\mathbf{r}_{1}, \sigma_{1}, \ldots, \mathbf{r}_{N}, \sigma_{N}\right) \\
& \quad=\xi^{3 N / 2} \Psi\left(\xi \mathbf{r}_{1}, \sigma_{1}, \ldots, \xi \mathbf{r}_{N}, \sigma_{N} ;[\mathrm{v}, \mathbf{A}]\right) .
\end{aligned}
$$

Since $\Psi_{\xi}$ is a normalized wave function and for $\xi=1$ it coincides with the GS function of the original system, Eq. (2.19), it can be used as a trial function for determination of the GS energy of this system by means of the Rayleigh-Ritz variational principle:

$$
E[\mathbf{v}, \mathbf{A}]=\min _{\xi}\left\langle\Psi_{\xi}|\hat{H}[\mathbf{v}, \mathbf{A}]| \Psi_{\xi}\right\rangle=\left.\langle\cdots\rangle\right|_{\xi=1} .
$$

Therefore, the necessary condition for this minimum must hold:

$$
0=\left.\frac{\partial}{\partial \xi}\left\langle\Psi_{\xi}|\hat{H}[\mathbf{v}, \mathbf{A}]| \Psi_{\xi}\right\rangle\right|_{\xi=1} .
$$

In terms of $\rho_{\xi, 1}, \ldots, n_{\xi, 2}-$ the DMs derived, according to Eqs. (2.14)-(2.18), from the wave function $\Psi_{\xi}-$ Eq. (2.21), the last equation (2.23) is rewritten with the help of Eq. (2.4) as

$$
\begin{aligned}
0= & \frac{\partial}{\partial \xi}\left\{\widetilde{T}_{0}\left[\rho_{\xi, 1}\right]+\widetilde{T}_{1}\left[\rho_{\xi, 1}, \mathbf{A}\right]+\widetilde{T}_{2}\left[n_{\xi}, \mathbf{A}^{2}\right]+\widetilde{T}_{1},\left[\gamma_{\xi, 1}, \mathbf{A}\right]\right. \\
& \left.+\widetilde{V}\left[n_{\xi}, \mathrm{V}\right]+\widetilde{E}_{\mathrm{ee}}\left[n_{\xi, 2}, u\right]\right\}_{\xi=1} .
\end{aligned}
$$

Then, by taking explicit form of $\xi$-dependent DMs and their functionals, e.g., $\mathbf{j}_{\mathrm{p}}\left(\mathbf{r} ;\left[\rho_{\xi, 1}\right]\right)=\xi^{4} \mathbf{j}_{\mathrm{p}}\left(\xi \mathbf{r} ;\left[\rho_{1}[\mathrm{v}, \mathbf{A}]\right]\right)$, and, next, by renaming variables of integration $\xi \mathbf{r} \rightarrow \mathbf{r}$, we find

$$
\begin{aligned}
0= & \frac{\partial}{\partial \xi}\left\{\xi^{2} \widetilde{T}_{0}\left[\rho_{1}[\mathrm{v}, \mathbf{A}]\right]+\xi \widetilde{T}_{1}\left[\rho_{1}[\mathrm{v}, \mathbf{A}], \mathbf{A}_{1 / \xi}\right]\right. \\
& +\widetilde{T}_{2}\left[n[\mathrm{v}, \mathbf{A}], \mathbf{A}_{1 / \xi}^{2}\right]+\xi \widetilde{T}_{1},\left[\gamma_{1}[\mathbf{v}, \mathbf{A}], \mathbf{A}_{1 / \xi}\right] \\
& \left.+\widetilde{V}\left[n[\mathbf{v}, \mathbf{A}], \mathbf{v}_{1 / \xi}\right]+\xi \widetilde{E}_{\mathrm{ee}}\left[n_{2}[\mathbf{v}, \mathbf{A}], u\right]\right\}_{\xi=1},
\end{aligned}
$$

where the modified fields are defined by

$$
\mathrm{v}_{\lambda}(\mathbf{r})=\mathrm{v}(\lambda \mathbf{r}), \quad \mathbf{A}_{\lambda}(\mathbf{r})=\mathbf{A}(\lambda \mathbf{r}) .
$$

The scaling $u\left(\xi^{-1} \mathbf{r}_{1}, \xi^{-1} \mathbf{r}_{2}\right)=\xi u\left(\mathbf{r}_{1}, \mathbf{r}_{2}\right)$ of the Coulombic potential, Eq. (2.3), was taken into account. After performing differentiations in Eq. (2.25) and changing the variable $\xi$ $\rightarrow \lambda=1 / \xi$, we obtain

$$
\begin{aligned}
\frac{\partial}{\partial \lambda} & \left\{\widetilde{T}_{1}\left[\rho_{1}[\mathrm{v}, \mathbf{A}], \mathbf{A}_{\lambda}\right]+\widetilde{T}_{2}\left[n[\mathrm{~V}, \mathbf{A}], \mathbf{A}_{\lambda}^{2}\right]+\widetilde{T}_{1^{\prime}}\left[\gamma_{1}[\mathrm{~V}, \mathbf{A}], \mathbf{A}_{\lambda}\right]\right. \\
& \left.+\widetilde{V}\left[n[\mathrm{v}, \mathbf{A}], \mathbf{v}_{\lambda}\right]\right\}_{\lambda=1} \\
& =2 T_{0}[\mathrm{v}, \mathbf{A}]+T_{1}[\mathbf{V}, \mathbf{A}]+T_{1},[\mathbf{v}, \mathbf{A}]+E_{\mathrm{ee}}[\mathbf{v}, \mathbf{A}] .
\end{aligned}
$$

Using Eq. (2.4), we can rewrite the left-hand side of Eq. (2.27) as a derivative of the expectation value of a modified Hamiltonian,

$$
\begin{aligned}
\frac{\partial}{\partial \lambda} & \left.\left\langle\Psi[\mathrm{v}, \mathbf{A}]\left|\hat{H}\left[\mathrm{v}_{\lambda}, \mathbf{A}_{\lambda}\right]\right| \Psi[\mathrm{v}, \mathbf{A}]\right\rangle\right|_{\lambda=1} \\
& =2 T_{0}[\mathrm{v}, \mathbf{A}]+T_{1}[\mathrm{v}, \mathbf{A}]+T_{1},[\mathrm{v}, \mathbf{A}]+E_{\mathrm{ee}}[\mathrm{v}, \mathbf{A}] .
\end{aligned}
$$

\section{Hellmann-Feynman theorem}

By virtue of the Hellmann-Feynman theorem, we have

$$
\begin{aligned}
\frac{\partial}{\partial \lambda} E & {\left.\left[\mathrm{v}_{\lambda}, \mathbf{A}_{\lambda}\right]\right|_{\lambda=1} } \\
& =\left\langle\Psi\left[\mathrm{v}_{1}, \mathbf{A}_{1}\right]\left|\frac{\partial}{\partial \lambda} \hat{H}\left[\mathrm{v}_{\lambda}, \mathbf{A}_{\lambda}\right]_{\lambda=1}\right| \Psi\left[\mathrm{v}_{1}, \mathbf{A}_{1}\right]\right\rangle .
\end{aligned}
$$

So, using this identity and also Eq. (2.20) we obtain from Eq. (2.28) the following VE:

$$
\left.\frac{\partial}{\partial \lambda} E\left[\mathrm{v}_{\lambda}, \mathbf{A}_{\lambda}\right]\right|_{\lambda=1}=E[\mathrm{v}, \mathbf{A}]+T_{0}[\mathrm{v}, \mathbf{A}]-T_{2}[\mathrm{v}, \mathbf{A}]-V[\mathrm{v}, \mathbf{A}] .
$$

The VE (2.30) is gauge invariant, because this property holds obviously for the total energy $E$ and the external scalar potential energy $V$, while for the combination $T_{0}-T_{2}$ it is proved in Appendix A.

The left-hand side of Eq. (2.30) - the change of the total energy due to the uniform scaling of the external fields, Eq. (2.26) - can be evaluated by means of functional differentiations, leading to another form of the VE:

$$
\begin{aligned}
\int & d^{3} r\left\{\frac{\widetilde{\delta} E[\mathrm{~V}, \mathbf{A}]}{\widetilde{\delta} \mathrm{V}(\mathbf{r})} \mathrm{V}^{\prime}(\mathbf{r})+\frac{\widetilde{\delta} E[\mathrm{~V}, \mathbf{A}]}{\widetilde{\delta} \mathbf{A}(\mathbf{r})} \cdot \mathbf{A}^{\prime}(\mathbf{r})\right\} \\
= & E+T_{0}-T_{2}-V,
\end{aligned}
$$

where $\widetilde{\delta}$ indicates the partial functional differentiation, and $\mathbf{V}^{\prime}$ and $\mathbf{A}^{\prime}$ are defined by [see Eq. (2.26)]

$f^{\prime}(\mathbf{r})=\left.\frac{\partial}{\partial \lambda} f(\lambda \mathbf{r})\right|_{\lambda=1}=\mathbf{r} \cdot \nabla(\mathbf{r}) f(\mathbf{r})=r \frac{\partial f(r, \theta, \varphi)}{\partial r}$,

with the last form written in the spherical coordinates.

To explore further the VE (2.31), we need to know the functional derivatives $\widetilde{\delta} E / \widetilde{\delta}$ v and $\widetilde{\delta} E / \widetilde{\delta} \mathbf{A}$. The way leading to explicit expressions for them will be demonstrated in detail in the example of the simpler case of $\delta \mathrm{v}$. By using Eq. (2.4) and the Hellmann-Feynman theorem, we have 


$$
\begin{aligned}
& \int d^{3} r \frac{\widetilde{\delta} E[\mathrm{v}, \mathbf{A}]}{\widetilde{\delta} \mathrm{v}(\mathbf{r})} \delta \mathrm{v}(\mathbf{r}) \\
& =\frac{d}{d \varepsilon} E[\mathrm{v}+\varepsilon \delta \mathrm{v}, \mathbf{A}]_{\varepsilon=0} \\
& =\left\langle\Psi[\mathrm{v}, \mathbf{A}]\left|\frac{d}{d \varepsilon} \hat{H}[\mathrm{v}+\varepsilon \delta \mathrm{v}, \mathbf{A}]_{\varepsilon=0}\right| \Psi[\mathrm{v}, \mathbf{A}]\right\rangle \\
& =\frac{d}{d \varepsilon} \widetilde{V}[n, \mathrm{v}+\varepsilon \delta \mathrm{v}]_{\varepsilon=0}=\widetilde{V}[n, \delta \mathrm{v}]
\end{aligned}
$$

[see, e.g., Eq. (A.2) of Ref. [14] for the definition of a functional derivative by means of first equality here], so

$$
\frac{\widetilde{\delta} E[\mathrm{~V}, \mathbf{A}]}{\widetilde{\delta} \mathrm{V}(\mathbf{r})}=n(\mathbf{r} ;[\mathrm{V}, \mathbf{A}])
$$

Similarly,

$$
\begin{aligned}
\frac{d}{d \varepsilon} E[\mathbf{v}, \mathbf{A}+\varepsilon \delta \mathbf{A}]_{\varepsilon=0}= & \widetilde{T}_{1}\left[\rho_{1}, \delta \mathbf{A}\right]+\widetilde{T}_{2}[n, 2 \mathbf{A} \cdot \delta \mathbf{A}] \\
& +\widetilde{T}_{1},\left[\gamma_{1}, \delta \mathbf{A}\right] .
\end{aligned}
$$

Therefore

$$
\begin{aligned}
\frac{\widetilde{\delta} E[\mathbf{v}, \mathbf{A}]}{\widetilde{\delta} \mathbf{A}(\mathbf{r})}= & e \mathbf{j}_{\mathrm{p}}\left(\mathbf{r} ;\left[\rho_{1}\right]\right)+2 n(\mathbf{r}) \frac{e^{2}}{2 m} \mathbf{A}(\mathbf{r}) \\
& -\left\{\boldsymbol{\nabla}(\mathbf{r}) \times \mathbf{m}\left(\mathbf{r} ;\left[\gamma_{1}\right]\right)\right\} .
\end{aligned}
$$

After inserting the results (2.34), (2.36), and (2.32) into Eq. (2.31), and using again Eqs. (2.4)-(2.8), we find the VE in the following form:

$$
\begin{aligned}
2 T_{0}+ & E_{\mathrm{ee}}-\int d^{3} r n(\mathbf{r})\{\mathbf{r} \cdot \boldsymbol{\nabla}(\mathbf{r})\}\left\{\mathbf{v}(\mathbf{r})+\frac{e^{2}}{2 m} \mathbf{A}^{2}(\mathbf{r})\right\} \\
& -\int d^{3} r e \mathbf{j}_{\mathrm{p}}\left(\mathbf{r} ;\left[\rho_{1}\right]\right) \cdot\{\mathbf{r} \cdot \boldsymbol{\nabla}(\mathbf{r})-1\} \mathbf{A}(\mathbf{r}) \\
& +\int d^{3} r \mathbf{m}\left(\mathbf{r} ;\left[\gamma_{1}\right]\right) \cdot\{\mathbf{r} \cdot \boldsymbol{\nabla}(\mathbf{r})\} \mathbf{B}(\mathbf{r},[\mathbf{A}])=0 .
\end{aligned}
$$

The following identity was helpful for obtaining the B-dependent term:

$$
\begin{aligned}
\int d^{3} r\{(\mathbf{r} \cdot \boldsymbol{\nabla}(\mathbf{r})) \mathbf{A}(\mathbf{r})\} \cdot\{\boldsymbol{\nabla}(\mathbf{r}) \times \mathbf{m}(\mathbf{r})\} \\
=\int d^{3} r \mathbf{m}(\mathbf{r}) \cdot\{1+\mathbf{r} \cdot \boldsymbol{\nabla}(\mathbf{r})\} \mathbf{B}(\mathbf{r},[\mathbf{A}]) .
\end{aligned}
$$

If the B-dependent term is neglected, Eq. (2.37) happens to be the same as the VE (36) in Ref. [16], obtained by Erhard and Gross, and as Eq. (2.20) in Ref. [15], obtained by us in a different way. The present VE (2.37) extends the above-mentioned investigations by including the
B-dependent term, which stems from the corresponding term (in its the most general form) in the Hamiltonian (2.2) considered here. It is worth noting that the B-dependent term in Eq. (2.37) vanishes for a homogeneous MF (B independent of $\mathbf{r})$.

\section{MOLECULE IN AN ARBITRARY MAGNETIC FIELD}

We are going now to specify the external scalar potential field to be created solely by $M$ nuclei held clamped at the positions $\mathbf{R}_{l}$ and having atomic numbers $Z_{l}$ :

$$
\mathrm{v}(\mathbf{r})=\mathrm{v}_{\mathrm{en}}(\mathbf{r} ; \mathbf{Z}, \mathbf{R})=-\frac{e^{2}}{4 \pi \varepsilon_{0}} \sum_{l=1}^{M} \frac{Z_{l}}{\left|\mathbf{r}-\mathbf{R}_{l}\right|},
$$

where the arrays of the potential characteristics are denoted as

$$
\begin{gathered}
\mathbf{R}=\left\{\mathbf{R}_{1}, \mathbf{R}_{2}, \ldots, \mathbf{R}_{M}\right\}=\left(R_{1 x}, R_{1 y}, \ldots, R_{M y}, R_{M z}\right), \\
\mathbf{Z}=\left\{Z_{1}, Z_{2}, \ldots, Z_{M}\right\} .
\end{gathered}
$$

So, the $N$-electron system now represents a molecule, which is neutral if $N=\Sigma_{l} Z_{l}$, or ionized, if $N$ is different.

From Eq. (3.1) it is easy to evaluate the derivative of the scaled potential [see Eq. (2.32)]:

$$
\begin{aligned}
\mathbf{v}_{e n}^{\prime}(\mathbf{r} ; \mathbf{Z}, \mathbf{R}) & =\frac{\partial}{\partial \lambda}\left\{\lambda^{-1} \mathbf{v}_{\mathrm{en}}\left(\mathbf{r} ; \mathbf{Z}, \lambda^{-1} \mathbf{R}\right)\right\}_{\lambda=1} \\
& =-\left(1+\mathbf{R} \cdot \frac{\partial}{\partial \mathbf{R}}\right) \mathbf{v}_{\mathrm{en}}(\mathbf{r} ; \mathbf{Z}, \mathbf{R})
\end{aligned}
$$

So, with the help of Eq. (2.34), the corresponding term in Eq. (2.31) in the case of a molecule can be written in the following form:

$$
\int d^{3} r \frac{\widetilde{\delta} E[\mathrm{v}, \mathbf{A}]}{\widetilde{\delta} \mathrm{v}(\mathbf{r})} \mathrm{v}^{\prime}(\mathbf{r})=-E_{\mathrm{en}}-\mathbf{R} \cdot \frac{\partial}{\partial \mathbf{R}} E\left[\mathrm{v}_{\mathrm{en}}(\cdot ; \mathbf{Z}, \mathbf{R}), \mathbf{A}\right],
$$

where the notation $f($ ) represents a function - the mapping $\mathbf{r} \rightarrow f(\mathbf{r})-$ and

$$
E_{\mathrm{en}}=\int d^{3} r n(\mathbf{r}) \mathrm{v}_{\mathrm{en}}(\mathbf{r})
$$

denotes the electron-nuclei interaction energy (it replaces the former term $V$ ). The Hellmann-Feynman theorem was used in the last step to transform the term involving $\partial \mathbf{v}_{\mathrm{en}} / \partial \mathbf{R}$ into $\langle\partial \hat{H} / \partial \mathbf{R}\rangle=\partial E / \partial \mathbf{R}$. Thus Eq. (3.5) transforms Eq. (2.31) into the following $\mathrm{VE}$ for a molecule:

$$
-\mathbf{R} \cdot \frac{\partial}{\partial \mathbf{R}} E+\int d^{3} r\left\{\frac{\widetilde{\delta} E\left[\mathrm{v}_{\mathrm{en}}, \mathbf{A}\right]}{\widetilde{\delta} \mathbf{A}(\mathbf{r})} \cdot \mathbf{A}^{\prime}(\mathbf{r})\right\}=E+T_{0}-T_{2} .
$$

It is convenient to consider the total energy of a molecule, $E_{\text {mol }}$, as a sum of the nucleus-nucleus interaction energy 


$$
E_{\mathrm{nn}}(\mathbf{Z}, \mathbf{R})=\frac{e^{2}}{4 \pi \varepsilon_{0}} \sum_{l<k}^{M} \frac{Z_{l} Z_{k}}{\left|\mathbf{R}_{l}-\mathbf{R}_{k}\right|},
$$

and the $N$-electron system energy $E$

$$
E_{\mathrm{mol}}=E_{\mathrm{nn}}+E=T+E_{\mathrm{C}},
$$

where

$$
T=T_{0}+T_{1}+T_{2}+T_{1}^{\prime}
$$

is the sum of all kinetic energy terms, and

$$
E_{\mathrm{C}}=E_{\mathrm{en}}+E_{\mathrm{ee}}+E_{\mathrm{nn}}
$$

is the sum of all Coulombic (C) energy contributions. Due to the Coulombic character of $E_{\mathrm{nn}}$, the following identity holds

$$
-\mathbf{R} \cdot \frac{\partial}{\partial \mathbf{R}} E_{\mathrm{nn}}(\mathbf{Z}, \mathbf{R})=-\left.\frac{\partial}{\partial \lambda} E_{\mathrm{nn}}(\mathbf{Z}, \lambda \mathbf{R})\right|_{\lambda=1}=+E_{\mathrm{nn}}(\mathbf{Z}, \mathbf{R}) .
$$

So, the sum of Eqs. (3.11) and (3.7) gives the following VE (note that $E_{\mathrm{nn}}$ is independent of $\mathbf{A}$ ):

$$
\begin{aligned}
-\mathbf{R} & \cdot \frac{\partial}{\partial \mathbf{R}} E_{\mathrm{mol}}+\int d^{3} r\left\{\frac{\widetilde{\delta} E_{\mathrm{mol}}\left[\mathrm{v}_{\mathrm{en}}, \mathbf{A}\right]}{\widetilde{\delta} \mathbf{A}(\mathbf{r})} \cdot \mathbf{A}^{\prime}(\mathbf{r})\right\} \\
& =E_{\mathrm{mol}}+T_{0}-T_{2},
\end{aligned}
$$

with $\widetilde{\delta} E_{\mathrm{mol}} / \widetilde{\delta} \mathbf{A} \equiv \widetilde{\delta} E / \widetilde{\delta} \mathbf{A}$ given in Eq. (2.36). The limits $\mathbf{A}^{\prime}$ $\rightarrow \mathbf{0}$ and $T_{1}, T_{2}, T_{1}, \rightarrow 0$ reduce this equation to the traditional VE for a molecule in the absence of a MF [see, e.g., Eq. (1.6.11) of Ref. [14]].

\section{MOLECULE IN A HOMOGENEOUS MAGNETIC FIELD}

\section{A. Virial equations}

Vector potentials for the homogeneous MF, $\mathbf{B}=$ const., are well known. Let us choose the following:

$$
\mathbf{A}(\mathbf{r})=\frac{1}{2} \mathbf{B} \times \mathbf{r}=\left(-\frac{1}{2} y, \frac{1}{2} x, 0\right) B, \quad \mathbf{B}=(0,0, B),
$$

where, without loss of generality, for the second form the $z$ axis of the coordinate system is chosen along the direction of B. The derivative, Eq. (2.32), of the scaled vector potential can be found immediately from Eq. (4.1) to be

$$
\mathbf{A}^{\prime}(\mathbf{r})=\frac{\partial}{\partial \lambda} \mathbf{A}(\lambda \mathbf{r})=\mathbf{A}(\mathbf{r})
$$

Therefore, with the help of Eq. (2.36), in the case of a homogeneous MF the corresponding term in Eq. (3.12) can be written in the following form [see Eqs. (2.6)-(2.8)]:

$$
\int d^{3} r\left\{\frac{\widetilde{\delta} E_{\mathrm{mol}}\left[\mathrm{v}_{\mathrm{en}}, \mathbf{A}\right]}{\widetilde{\delta} \mathbf{A}(\mathbf{r})} \cdot \mathbf{A}^{\prime}(\mathbf{r})\right\}=T_{1}+2 T_{2}+T_{1^{\prime}} .
$$

By inserting $\mathbf{V}$ in the form (3.1) and $\mathbf{A}$ in the form (4.1) into Eq. (2.2) we obtain the Hamiltonian $\hat{H}$ depending ex- plicitly on the arrays $\mathbf{Z}, \mathbf{R}$ and the scalar $B$; therefore, the molecular energy and all its components, Eq. (3.9), depend on the same arguments:

$$
E_{\mathrm{mol}}=E_{\mathrm{mol}}(\mathbf{Z}, \mathbf{R}, B)=T_{0}(\mathbf{Z}, \mathbf{R}, B)+\cdots,
$$

while the Hellmann-Feynman theorem in connection with such a Hamiltonian gives immediately

$$
B \frac{\partial}{\partial B} E_{\mathrm{mol}}(\mathbf{Z}, \mathbf{R}, B)=T_{1}+2 T_{2}+T_{1^{\prime}} .
$$

Thus, due to Eq. (4.3) and (4.5), the VE (3.12) can be rewritten as

$$
\left(-\mathbf{R} \cdot \frac{\partial}{\partial \mathbf{R}}+B \frac{\partial}{\partial B}-1\right) E_{\mathrm{mol}}=T_{0}-T_{2} .
$$

But, from Eqs. (4.5) and (3.9) we have

$$
\left(B \frac{\partial}{\partial B}-1\right) E_{\mathrm{mol}}=T_{2}-T_{0}-E_{\mathrm{C}} .
$$

The sum of Eq. (4.6) and (4.7) leads to the VE in an alternative form

$$
\left(-\mathbf{R} \cdot \frac{\partial}{\partial \mathbf{R}}+2 B \frac{\partial}{\partial B}-2\right) E_{\mathrm{mol}}=-E_{\mathrm{C}},
$$

and, next, using Eq. (3.9), to a form

$$
\left(-\mathbf{R} \cdot \frac{\partial}{\partial \mathbf{R}}+2 B \frac{\partial}{\partial B}\right) E_{\mathrm{mol}}=2 T+E_{\mathrm{C}} .
$$

\section{B. Uniform scaling of a molecule}

Although the term $\mathbf{R} \cdot \partial E_{\mathrm{mol}} / \partial \mathbf{R}$ in the VEs (4.6) and (4.8) involves $3 \mathrm{M}$ coordinates of the nuclear skeleton of a molecule, Eq. (3.2), it can be, in fact, expressed as a derivative with respect to a single parameter [compare Eq. (2.32)]:

$$
\mathbf{R} \cdot \frac{\partial}{\partial \mathbf{R}} E_{\mathrm{mol}}(\mathbf{Z}, \mathbf{R}, B)=\left.\frac{\partial}{\partial \lambda} E_{\mathrm{mol}}(\mathbf{Z}, \lambda \mathbf{R}, B)\right|_{\lambda=1} .
$$

This property suggests the formulation of a problem of the uniformly scaled molecule: we choose and fix some configuration of nuclear positions $\mathbf{R}^{0}$ [this includes also a particular orientation of a molecule with respect to $\mathbf{B}$, according to the convention in Eq. (4.1)] and, next, we consider a set of configurations $\mathbf{R}$ obtained from $\mathbf{R}^{0}$ by the uniform scaling with a dimensionless parameter $R$ :

$$
\mathbf{R}=R \mathbf{R}^{0}, \quad 0<R \leqslant \infty .
$$

We will call $R$ the size parameter of a molecule. It should be noted that each bond length is scaled in the same way, $\mid \mathbf{R}_{l}$ $-\mathbf{R}_{l^{\prime}}|=R| \mathbf{R}_{l}^{0}-\mathbf{R}_{l^{\prime}}^{0} \mid$, while bond angles and orientation angles remain unchanged during scaling. We are going to find the field dependence of the GS energy $E_{\mathrm{mol}}$ of the molecule characterized by Z, Eq. (3.3), and by R, Eq. (3.2), in the form Eq. (4.10). The energy of a highly squeezed molecule (of a very small size $R$ ) is very large due to the $E_{\mathrm{nn}}$ $\propto 1 / R$ term in Eq. (3.9), while the term $E$ tends to the finite 
energy of the "united atom" having $Z=\Sigma_{l} Z_{l}$. At infinite size parameter, the molecule dissociates into atoms. If the initial molecule was ionized, some of these atoms are ionized. Knowledge of the atomic energies together with the ionization potentials (electron affinities) of all atoms would be sufficient to determine the species which are the anions (cations), by looking for the minimum of the total energy of the system of atoms and ions.

From Eq. (4.9) we find

$$
\left.\mathbf{R} \cdot \frac{\partial}{\partial \mathbf{R}} E_{\mathrm{mol}}(\mathbf{Z}, \mathbf{R}, B)\right|_{\mathbf{R}=R \mathbf{R}^{0}}=R \frac{\partial}{\partial R} E_{\mathrm{mol}}\left(\mathbf{Z}, R \mathbf{R}^{0}, B\right) .
$$

Since now we simplify the notation: $E_{\text {mol }}\left(\mathbf{Z}, R \mathbf{R}^{0}, B\right)$ $\rightarrow E_{\mathrm{mol}}(R, B)$, and similarly for all components of $E_{\mathrm{mol}}$, Eq. (3.9), and for DMs, by suppressing the dependence on the fixed $\mathbf{Z}$ and $\mathbf{R}^{0}$. After inserting the result (4.11), the VEs (4.6) and (4.8) represent partial differential equations for $E_{\text {mol }}$ as a function of two independent variables $R, B$ :

$$
\left(-R \frac{\partial}{\partial R}+B \frac{\partial}{\partial B}-1\right) E_{\mathrm{mol}}(R, B)=T_{0 \overline{2}}(R, B),
$$

with $T_{0 \overline{2}}=T_{0}-T_{2}$, and

$$
\begin{aligned}
& \left(-\frac{1}{2} R \frac{\partial}{\partial R}+B \frac{\partial}{\partial B}-1\right) E_{\mathrm{mol}}(R, B)=-\frac{1}{2} E_{\mathrm{C}}(R, B) . \\
& \left(-R \frac{\partial}{\partial R}+2 B \frac{\partial}{\partial B}\right) E_{\mathrm{mol}}(R, B)=2 T(R, B)+E_{\mathrm{C}}(R, B) .
\end{aligned}
$$

The difference between Eqs. (4.12) and (4.13a) leads to the expression for the derivative of the molecular energy with respect to the size parameter:

$$
R \frac{\partial}{\partial R} E_{\mathrm{mol}}(R, B)=-\left\{E_{C}(R, B)+2 T_{02}(R, B)\right\}
$$

a counterpart of Eq. (4.5). At the optimum size $R_{\text {opt }}(B)$ for a given $B$, for which $E_{\text {mol }}(R, B)$ reaches minimum, so the equation

$$
\left.\frac{\partial}{\partial R} E_{\mathrm{mol}}(R, B)\right|_{R=R_{\mathrm{opt}}(B)}=0
$$

holds, we see from Eq. (4.14) that the following relation between the Coulombic and kinetic energy contributions must obey

$$
E_{\mathrm{C}}\left(R_{\text {opt }}(B), B\right)+2 T_{02}\left(R_{\text {opt }}(B), B\right)=0,
$$

valid at arbitrary $B$. It should be stressed that during minimization over $R$, Eq. (4.15), the remaining parameters of the molecule geometry, like ratios of bond lengths, bond angles, orientation angles, all are held fixed; therefore, they may not be at the optimum.

\section{C. $(R, B)$-dependent molecular energy in terms of 1DM}

With the help of Eqs. (2.5), (2.7), and (4.1), the right-hand side of Eq. (4.12) for the homogeneous MF can be written as

$$
\begin{aligned}
T_{0 \overline{2}}(R, B) & =T_{0}(R, B)-T_{2}(R, B) \\
& =\int d^{3} r\left\{t(\mathbf{r} ; R, B)-\frac{e^{2}}{8 m} n(\mathbf{r} ; R, B)\left(x^{2}+y^{2}\right) B^{2}\right\},
\end{aligned}
$$

i.e., in terms of the conventional kinetic-energy density $t(\mathbf{r})$ and the electron-number density $n(\mathbf{r})$, both derivable from the 1DM $\rho_{1}$, Eqs. (2.11) and (2.18). For small $B, T_{0 \overline{2}}(R, B)$ is positive, see Eq. (2.11). It should be recalled here that the separate terms $T_{0}$ and $T_{2}$ at $B \neq 0$ have no physical meaning as depending on a gauge; however, the combination $T_{0}$ $-T_{2}$ is gauge invariant (see Appendix A).

Let us suppose that the dependence of $\rho_{1}$ on $R$ and $B$ is known. So $T_{02}(R, B)$ is available and $E_{\text {mol }}(R, B)$ can be found by solving Eq. (4.12). It will be convenient to transform this equation by subtracting from it the $B \rightarrow 0$ limiting equation

$$
\left(-R \frac{\partial}{\partial R}+B \frac{\partial}{\partial B}-1\right) \delta E_{\mathrm{mol}}(R, B)=\delta T_{0 \overline{2}}(R, B),
$$

where

$$
\delta F(R, B)=F(R, B)-F(R, 0) .
$$

The unique solution $\delta E_{\text {mol }}$ of Eq. (4.18a), passing through the initial condition line $\delta E_{\text {mol }}^{\text {ini }}\left(R, B_{0}\right)$ (assumed to be a known function of $R$ for some fixed $B_{0}$ ) can be written in the form

$$
\begin{aligned}
\delta E_{\mathrm{mol}}(R, B)= & \left(B / B_{0}\right) \delta E_{\mathrm{mol}}^{\mathrm{ini}}\left(R B / B_{0}, B_{0}\right) \\
& +\int_{B_{0} / B}^{1} d \zeta \zeta^{-2} \delta T_{0}(R / \zeta, B \zeta) .
\end{aligned}
$$

It is easy to check directly that this solution satisfies the initial condition and Eq. (4.18a).

But, in fact, we would like to have for the initial condition the GS energy of a molecule in the absence of a MF. For small $B_{0}$, we can expand $E_{\mathrm{mol}}^{\mathrm{ini}}\left(R^{\prime}, B_{0}\right)$ in the Taylor series with respect to $B_{0}$, so the corresponding term of Eq. (4.19) is

$$
\begin{aligned}
\frac{B}{B_{0}} \delta E_{\mathrm{mol}}^{\mathrm{ini}}\left(R B / B_{0}, B_{0}\right) \\
=B\left\{\frac{\partial E_{\mathrm{mol}}^{\mathrm{ini}}\left(R^{\prime}, B^{\prime}\right)}{\partial B^{\prime}}+\frac{1}{2} B_{0} \frac{\partial^{2} E_{\mathrm{mol}}^{\mathrm{ini}}\left(R^{\prime}, B^{\prime}\right)}{\left(\partial B^{\prime}\right)^{2}}\right. \\
+\cdots\}_{B^{\prime}=0, R^{\prime}=R B / B_{0}} \cdot
\end{aligned}
$$

From Eq. (4.5), using Eqs. (4.1) and (2.6)-(2.8) we find 


$$
\begin{aligned}
\frac{\partial E_{\mathrm{mol}}^{\mathrm{ini}}\left(R^{\prime}, B^{\prime}\right)}{\partial B^{\prime}}= & \int d^{3} r\left\{\frac{1}{2}\left(\mathbf{r} \times \mathbf{j}_{\mathrm{p}}\left(\mathbf{r} ; R^{\prime}, B^{\prime}\right)\right)_{z}\right. \\
& +\frac{e^{2}}{4 m} B^{\prime} n\left(\mathbf{r} ; R^{\prime}, B^{\prime}\right)\left(x^{2}+y^{2}\right) \\
& \left.-m_{z}\left(\mathbf{r} ; R^{\prime}, B^{\prime}\right)\right\} .
\end{aligned}
$$

Let us note that at $B=0$ (and $\mathbf{A}=\mathbf{0}$ chosen), the Hamiltonian (2.2) is real, therefore its eigenfunction can be chosen real too, what leads to real $1 \mathrm{DM} \rho_{1}$ and then to $\mathbf{j}_{\mathrm{p}}(\mathbf{r})=\mathbf{0}$, according to Eq. (2.12), second line. Thus only the third term of Eq. (4.21) enters Eq. (4.20) at $B^{\prime}=0$. Finally, the limiting form of Eq. (4.19) for $B_{0} \rightarrow 0$ is

$$
\begin{aligned}
E_{\mathrm{mol}}(R, B)= & E_{\mathrm{mol}}(R, 0)-B \mathcal{M}_{z} \\
& +\int_{0}^{1} d \zeta \zeta^{-2}\left\{T_{0 \overline{2}}(R / \zeta, B \zeta)-T_{0 \overline{2}}(R / \zeta, 0)\right\},
\end{aligned}
$$

where, according to Eq. (2.13), the $z$ component of the magnetization vector for a molecule dissociated into atoms at $B$ $=0$ is

$$
\begin{aligned}
\mathcal{M}_{z} & =\lim _{R^{\prime} \rightarrow \infty} \int d^{3} r m_{z}\left(\mathbf{r} ; R^{\prime}, 0\right) \\
& =-\frac{g e \hbar}{4 m} \sum_{l=1}^{M}\left\{N_{\uparrow}\left(Z_{l}\right)-N_{\downarrow}\left(Z_{l}\right)\right\} .
\end{aligned}
$$

Here $N_{\sigma}\left(Z_{l}\right), \sigma=\uparrow, \downarrow$, is the number of electrons with the spin $\sigma$ in the atom (ion) characterized by $Z_{l}$. The integral in Eq. (4.22) must be convergent at lower limit, because all remaining terms of this equation are finite.

\section{D. $(R, B)$-dependent molecular energy in terms of pair density}

The total Coulombic energy $E_{C}(R, B)$, involved in the VE (4.13), can be written with the help of Eqs. (3.10b), (3.6), (3.1), (2.10), and (3.8), as

$$
\begin{aligned}
E_{\mathrm{C}}(R, B)= & \frac{e^{2}}{4 \pi \varepsilon_{0}}\left\{-\sum_{l=1}^{M} Z_{l} \int d^{3} r \frac{n(\mathbf{r} ; R, B)}{\left|\mathbf{r}-R \mathbf{R}_{l}^{0}\right|}\right. \\
& +\int d^{3} r_{1} d^{3} r_{2} \frac{n_{2}\left(\mathbf{r}_{1}, \mathbf{r}_{2} ; R, B\right)}{\left|\mathbf{r}_{1}-\mathbf{r}_{2}\right|} \\
& \left.+\frac{1}{R} \sum_{l<k}^{M} \frac{Z_{l} Z_{k}}{\left|\mathbf{R}_{l}^{0}-\mathbf{R}_{k}^{0}\right|}\right\},
\end{aligned}
$$

i.e., in terms of the electron-pair density $n_{2}\left(\mathbf{r}_{1}, \mathbf{r}_{2} ; R, B\right)$ and the electron-number density $n(\mathbf{r} ; R, B)$. The last can be obtained from the former [see Eqs. (2.15)-(2.18)]:

$$
n(\mathbf{r} ; R, B)=\frac{2}{N-1} \int d^{3} r^{\prime} n_{2}\left(\mathbf{r}, \mathbf{r}^{\prime} ; R, B\right)
$$

Let us suppose that the dependence of $n_{2}$ on $R$ and $B$ is known, so the function $E_{C}(R, B)$ is available for solving Eq. (4.13a). By applying steps analogous to these in Sec. IV C, we have the partial differential equation

$$
\left(-\frac{1}{2} R \frac{\partial}{\partial R}+B \frac{\partial}{\partial B}-1\right) \delta E_{\mathrm{mol}}(R, B)=-\frac{1}{2} \delta E_{\mathrm{C}}(R, B)
$$

and its solution

$$
\begin{aligned}
\delta E_{\mathrm{mol}}(R, B)= & \left(B / B_{0}\right) \delta E_{\mathrm{mol}}^{\mathrm{ini}}\left(R\left(B / B_{0}\right)^{1 / 2}, B_{0}\right) \\
& -(1 / 2) \int_{B_{0} / B}^{1} d \zeta \zeta^{-2} \delta E_{\mathrm{C}}\left(R / \zeta^{1 / 2}, B \zeta\right),
\end{aligned}
$$

passing through the initial condition line $\delta E_{\mathrm{mol}}^{\mathrm{ini}}\left(R, B_{0}\right)$. The $B_{0} \rightarrow 0$ limit of this result is obtained in the same way as previously:

$$
\begin{aligned}
E_{\mathrm{mol}}(R, B)= & E_{\mathrm{mol}}(R, 0)-B \mathcal{M}_{z} \\
& -\frac{1}{2} \int_{0}^{1} d \zeta \zeta^{-2}\left\{E_{\mathrm{C}}\left(R / \zeta^{1 / 2}, B \zeta\right)-E_{\mathrm{C}}\left(R / \zeta^{1 / 2}, 0\right)\right\},
\end{aligned}
$$

where $\mathcal{M}_{z}$ is given in Eq. (4.23). The integral must be convergent because all remaining terms in the equation are finite.

By equating two solutions (4.22) and (4.28) we find the following relation, valid at arbitrary $R$ and $B$ :

$$
\begin{aligned}
& \int_{0}^{1} d \zeta \zeta^{-2}\left\{\left[E_{\mathrm{C}}\left(R / \zeta^{1 / 2}, B \zeta\right)-E_{\mathrm{C}}\left(R / \zeta^{1 / 2}, 0\right)\right]\right. \\
& \left.\quad+2\left[T_{0 \overline{2}}(R / \zeta, B \zeta)-T_{02}(R / \zeta, 0)\right]\right\}=0
\end{aligned}
$$

which generalizes the relation (4.16) valid at the optimum size parameter.

\section{E. $B$-dependent molecular energy at fixed geometry}

Equation (4.7) can be considered a differential equation for $B$ dependence of $E_{\text {mol }}$ when the geometry of a molecule, $\mathbf{R}=R \mathbf{R}^{0}$, is kept fixed. After subtracting from it the $B \rightarrow 0$ limiting equation we have

$$
\left(B \frac{\partial}{\partial B}-1\right) \delta E_{\mathrm{mol}}(R, B)=\delta E_{\mathrm{fd}}(R, B),
$$

where we denoted by $E_{\mathrm{fd}}$ the combination of energy terms determining the field dependence (fd):

$$
E_{\mathrm{fd}}=T_{2}-T_{0}-E_{\mathrm{C}}=-\left(T_{0 \overline{2}}+E_{\mathrm{C}}\right),
$$

see Eqs. (4.17) and (4.24) for the explicit form. The solution of Eq. (4.30) passing through the initial condition $\delta E_{\mathrm{mol}}^{\mathrm{ini}}\left(R, B_{0}\right)$ is 


$$
\begin{aligned}
\delta E_{\mathrm{mol}}(R, B)= & \left(B / B_{0}\right) \delta E_{\mathrm{mol}}^{\mathrm{ini}}\left(R, B_{0}\right) \\
& +\int_{B_{0} / B}^{1} d \zeta \zeta^{-2} \delta E_{\mathrm{fd}}(R, B \zeta) .
\end{aligned}
$$

Using Eqs. (4.20) and (4.21), we find the $B_{0} \rightarrow 0$ limit of the solution

$$
\begin{aligned}
E_{\mathrm{mol}}(R, B)= & E_{\mathrm{mol}}(R, 0)-B \mathcal{M}_{z}(R) \\
& +\int_{0}^{1} d \zeta \zeta^{-2}\left\{E_{\mathrm{fd}}(R, B \zeta)-E_{\mathrm{fd}}(R, 0)\right\},
\end{aligned}
$$

where $\mathcal{M}_{z}(R)$ is the magnetization vector of a molecule at $B=0$ :

$$
\mathcal{M}_{z}(R)=-\frac{g e \hbar}{4 m}\left[N_{\uparrow}\left(\mathbf{Z}, R \mathbf{R}^{0}\right)-N_{\downarrow}\left(\mathbf{Z}, R \mathbf{R}^{0}\right)\right]
$$

Here $N_{\sigma}\left(\mathbf{Z}, R \mathbf{R}^{0}\right)$ is the number of electrons with the spin $\sigma$ in the molecule characterized by $\mathbf{Z}, R \mathbf{R}^{0}$ [compare Eq. (4.23)].

\section{F. Extrapolating field dependence}

The expressions for $E_{\mathrm{mol}}(R, B)$ obtained in the previous Secs. IV C, IV D, and IV E involve, in addition to the information at $B=0$, the functions $T_{0 \overline{2}}, E_{\mathrm{C}}$, and $E_{\mathrm{fd}}$ from a wide range of arguments $R^{\prime}$ and $B^{\prime}$. Such information may be difficult to find; therefore, it may prove useful to have expressions extrapolating to the field $B$ the results known at the field $B_{0}$ by using the mentioned functions from a narrower range of arguments. Such expressions are easy to obtain from Eqs. (4.18a), (4.26), and (4.30) when $\delta$ is omitted both in the equations and in their solutions, and the substitution $E_{\mathrm{mol}}^{\mathrm{ini}} \rightarrow E_{\mathrm{mol}}$ is applied:

$$
\begin{gathered}
E_{\mathrm{mol}}(R, B)=\left(B / B_{0}\right) E_{\mathrm{mol}}\left(R B / B_{0}, B_{0}\right) \\
+\int_{B_{0} / B}^{1} d \zeta \zeta^{-2} T_{0}(R / \zeta, B \zeta), \\
E_{\mathrm{mol}}(R, B)=\left(B / B_{0}\right) E_{\mathrm{mol}}\left(R\left(B / B_{0}\right)^{1 / 2}, B_{0}\right) \\
-(1 / 2) \int_{B_{0} / B}^{1} d \zeta \zeta^{-2} E_{\mathrm{C}}\left(R / \zeta^{1 / 2}, B \zeta\right), \\
E_{\mathrm{mol}}(R, B)=\left(B / B_{0}\right) E_{\mathrm{mol}}\left(R, B_{0}\right)+\int_{B_{0} / B}^{1} d \zeta \zeta^{-2} E_{\mathrm{fd}}(R, B \zeta) .
\end{gathered}
$$

For the solution in terms of $T_{0 \overline{2}}$ or $E_{\mathrm{C}}$, separately, the extrapolation starts from the molecular energy at the field $B_{0}$ and at the size $R_{0}=R B / B_{0}$ or $R_{0}=R\left(B / B_{0}\right)^{1 / 2}$, and involves functions with the arguments in the range $R \leqslant R^{\prime} \leqslant R_{0}$ and $B_{0} \leqslant B^{\prime} \leqslant B$, while for the solution in terms of $E_{\mathrm{fd}}$, there is $R=R^{\prime}=R_{0}$.

\section{ATOM IN A HOMOGENEOUS MAGNETIC FIELD}

For an atom, the electron-nuclei potential $\mathrm{v}_{\mathrm{en}}$, Eq. (3.1), reduces to one term $\left(M=1, Z_{1}=Z\right)$ :

$$
\mathrm{v}_{\mathrm{en}}(\mathbf{r} ; Z)=-\frac{e^{2}}{4 \pi \varepsilon_{0}} \frac{Z}{|\mathbf{r}|} \text {. }
$$

We have chosen for its position vector $\mathbf{R}_{1}=\mathbf{0}$, because in a homogeneous MF the atomic energy can not depend on the position of an atom. It depends, in fact, on three scalar parameters only, $E=E(N, Z, B)$. The dependence on $Z$ enters the expectation value (2.4) of the atomic Hamiltonian via the term connected with $\mathrm{v}_{\mathrm{en}}$, Eq. (5.1),

$$
\widetilde{V}[n, \mathrm{v}]=\widetilde{E}_{\mathrm{en}}[n, Z]=\int d^{3} r n(\mathbf{r}) \mathrm{v}_{\mathrm{en}}(\mathbf{r} ; Z)
$$

So, by applying the Hellmann-Feynman identity we can find immediately for the $Z$ dependence

$$
\begin{aligned}
Z \frac{\partial}{\partial Z} E(N, Z, B) & =\left.Z \frac{\partial}{\partial Z^{\prime}} \widetilde{E}_{\mathrm{en}}\left[n(. ; N, Z, B), Z^{\prime}\right]\right|_{Z^{\prime}=Z} \\
& =E_{\mathrm{en}}(N, Z, B) .
\end{aligned}
$$

When adapting molecular results of the preceding section to the atomic case, we replace $E_{\text {mol }}$ by $E$, because $E_{\mathrm{nn}}$ is absent, Eqs. (3.8), (3.9), and we remove any reference to $R$. We also simplify the notation: $E(N, Z, B) \rightarrow E(B)$, and similarly for all components of $E$, by suppressing the dependence on $N$ and $Z$, which are kept fixed for the relations written below. Thus from Eq. (4.5) we have

$$
B \frac{\partial}{\partial B} E(B)=T_{1}+2 T_{2}+T_{1}
$$

for the derivative with respect to $B$, and from Eqs. (4.12) and (4.13)

$$
\begin{gathered}
\left(B \frac{\partial}{\partial B}-1\right) E(B)=T_{0 \overline{2}}(B), \\
\left(B \frac{\partial}{\partial B}-1\right) E(B)=-\frac{1}{2} E_{\mathrm{C}}(B), \\
2 B \frac{\partial}{\partial B} E(B)=2 T(B)+E_{\mathrm{C}}(B)
\end{gathered}
$$

for the VEs. The Coulombic energy for an atom is limited to $E_{\mathrm{C}}=E_{\mathrm{ee}}+E_{\mathrm{en}}$. The difference of Eqs. (5.5) and (5.6a) gives the relation

$$
E_{\mathrm{C}}(B)+2 T_{02}(B)=0,
$$

valid for arbitrary $B$ [the analogous relation for a molecule, Eq. (4.16), holds at the equilibrium size]. The $B \rightarrow 0$ limit of Eq. (5.7) or (5.6b) is equivalent to the well known relation for an atom in the absence of a MF (see, e.g., Ref. [14])

$$
\left\langle\hat{V}_{\mathrm{en}}+\hat{V}_{\mathrm{ee}}\right\rangle+2\langle\hat{T}\rangle=0 .
$$


The solutions (4.22) and (4.28) of VEs for molecules, adapted now for atoms, are

$$
\begin{aligned}
& E(B)=E(0)-B \mathcal{M}_{z}+\int_{0}^{1} d \zeta \zeta^{-2}\left\{T_{0 \overline{2}}(B \zeta)-T_{0 \overline{2}}(0)\right\}, \\
& E(B)=E(0)-B \mathcal{M}_{z}-\frac{1}{2} \int_{0}^{1} d \zeta \zeta^{-2}\left\{E_{\mathrm{C}}(B \zeta)-E_{\mathrm{C}}(0)\right\},
\end{aligned}
$$

where $\mathcal{M}_{z}$ is given by Eq. (4.23) limited to one term only. Equivalence of the two forms of the atomic energy, Eqs. (5.9) and (5.10), is obvious from Eq. (5.7). The convergence of the integral can be proven from the VE (5.5): after inserting there the series for $E(B)$ and $T_{0 \overline{2}}(B)$ expanded in powers of $B$, we find from the linear terms that $\left\{\partial T_{0 \overline{2}}(B) / \partial B\right\}_{B=0}$ $=0$, so for $\zeta \rightarrow 0$ the integrand tends to the finite value $\frac{1}{2} B^{2}\left\{\partial^{2} T_{0} \overline{2}\left(B^{\prime}\right) /\left(\partial B^{\prime}\right)^{2}\right\}_{B^{\prime}=0}$.

All three extrapolating expressions (4.35)-(4.37) for a molecule give one expression when adapted for an atom:

$$
E(B)=\left(B / B_{0}\right) E\left(B_{0}\right)+\int_{B_{0} / B}^{1} d \zeta \zeta^{-2} T_{02}(B \zeta) .
$$

\section{FIELD DEPENDENCE OF APPROXIMATE GROUND-STATE SOLUTIONS AND OF EXCITED-STATE SOLUTIONS}

\section{A. Variational solutions}

All results, obtained in the previous sections, concern the exact GS energy and DMs, as corresponding to the GS solution of the Schrödinger equation (2.19). No approximations were involved during the derivations. It is interesting that these results hold also for some approximate solutions. We discuss now the solutions obtained from the variational principle

$$
\begin{aligned}
E[\mathrm{v}, \mathbf{A}] & \leqslant E^{\Omega}[\mathrm{v}, \mathbf{A}]=\min _{\Psi \in \Omega}\langle\Psi|\hat{H}[\mathrm{v}, \mathbf{A}]| \Psi\rangle \\
& =\left.\langle\cdots\rangle\right|_{\Psi=\Psi^{\Omega}[\mathrm{v}, \mathbf{A}]} .
\end{aligned}
$$

Here we denoted by $\Omega$ a particular set of normalized $N$-electron trial wave functions. Used as the superscript, $\Omega$ indicates the approximate GS solution defined by Eq. (6.1). Thus $\left\{E^{\Omega}[\mathbf{v}, \mathbf{A}], \Psi^{\Omega}[\mathbf{v}, \mathbf{A}]\right\}$ of Eq. (6.1) will play the role analogous to $\{E[\mathbf{v}, \mathbf{A}], \Psi[\mathbf{v}, \mathbf{A}]\}$, the solution of Eq. (2.19). The most important example of $\Omega$ is a set of Slater determinants, leading, via Eq. (6.1), to the unrestricted Hartree-Fock results. A wider set may define each trial wave function to be a linear combination of a few Slater determinants, exhibiting some particular (e.g., spin) symmetry. There is no need to give further examples. The only requirement for $\Omega$ to be applicable for our purposes is that the relation

$$
\Psi_{\xi}^{\Omega} \in \Omega
$$

must be true. Here $\Psi_{\xi}^{\Omega}$ denotes the solution $\Psi^{\Omega}[\mathrm{v}, \mathbf{A}]$ transformed by means of the scaling defined in Eq. (2.21). The property (6.2) is fulfilled by a general Hartree-Fock set and by a general multideterminantal set (however, not in the re- stricted cases for which any molecular orbital of a Slater determinant is represented as linear combination of a finite number of fixed basis functions with the coefficients playing the role of variational parameters). When Eq. (6.2) is true, Eqs. (2.22)-(2.28) hold for $\left\{E^{\Omega}, \Psi^{\Omega}\right\}$.

The Hellmann-Feynman theorem was invoked many times in the preceding sections. It is easy to verify that it holds not only for the solution $\{E[\mathbf{v}, \mathbf{A}], \Psi[\mathbf{v}, \mathbf{A}]\}$ of the Schrödinger equation (2.19), but also for the variational solution $\left\{E^{\Omega}[\mathbf{v}, \mathbf{A}], \Psi^{\Omega}[\mathbf{v}, \mathbf{A}]\right\}$ of Eq. (6.1). To see this, let us consider the Hamiltonian $\hat{H}(\epsilon)$ which depends on a parameter $\epsilon$, so the variational solution, corresponding to it, depends on $\epsilon$, too:

$$
E^{\Omega}(\epsilon)=\min _{\Psi \in \Omega}\langle\Psi|\hat{H}(\epsilon)| \Psi\rangle=\left.\langle\cdots\rangle\right|_{\Psi=\Psi^{\Omega}(\epsilon)}
$$

Now, applying the rules of differentiation to this solution, we find

$$
\begin{aligned}
\frac{d}{d \epsilon} E^{\Omega}(\epsilon)= & \frac{d}{d \epsilon}\left\langle\Psi^{\Omega}(\epsilon)|\hat{H}(\epsilon)| \Psi^{\Omega}(\epsilon)\right\rangle \\
= & \left.\frac{d}{d \epsilon^{\prime}}\left\langle\Psi^{\Omega}(\epsilon)\left|\hat{H}\left(\epsilon^{\prime}\right)\right| \Psi^{\Omega}(\epsilon)\right\rangle\right|_{\epsilon^{\prime}=\epsilon} \\
& +\left.\frac{d}{d \epsilon^{\prime}}\left\langle\Psi^{\Omega}\left(\epsilon^{\prime}\right)|\hat{H}(\epsilon)| \Psi^{\Omega}\left(\epsilon^{\prime}\right)\right\rangle\right|_{\epsilon^{\prime}=\epsilon} .
\end{aligned}
$$

But the second term of the last result vanishes. To prove this we use the function $\Psi^{\Omega}\left(\epsilon^{\prime}\right) \in \Omega$ as a trial function in the minimization (6.3): the minimum with respect to $\epsilon^{\prime}$ is reached at $\epsilon^{\prime}=\epsilon$. So, finally, Eq. (6.4) can be rewritten as

$$
\frac{d}{d \epsilon} E^{\Omega}(\epsilon)=\left\langle\Psi^{\Omega}(\epsilon)\left|\frac{d \hat{H}(\epsilon)}{d \epsilon}\right| \Psi^{\Omega}(\epsilon)\right\rangle,
$$

i.e., in the traditional form of the Hellmann-Feynman identity.

Thus all results obtained in Secs. II-V for the exact GS solution hold also for all those variational solutions $E^{\Omega}[\mathrm{v}, \mathbf{A}], \Psi^{\Omega}[\mathrm{v}, \mathbf{A}]$, for which the set $\Omega$ of trial functions shows the property (6.2). In particular, the discrepancy in the satisfaction of the VE (5.7) for atoms and Eqs. (4.14) or (4.16) for molecules may be used for checking the numerical accuracy (convergence) of the Hartree-Fock calculations, similarly as the relation (5.8) is widely used for this purpose for atoms in the absence of a MF.

\section{B. Excited-state solutions}

One can define the set $\Omega$ of trial functions used in Eq. (6.1) as the subspace of the Hilbert space of normalized $N$-electron functions, which consists of all functions having particular symmetry properties (i.e., which transform as rows of a particular irreducible representation of the group of the Schrödinger equation for the Hamiltonian $\hat{H}$ ). If this symmetry is different from the symmetry of the GS, the solution $E^{\Omega}$ of Eq. (6.1) represents the exact excited energy, the lowest 
one of this symmetry. However, when the definition of $\Omega$ imposes some restrictions (model forms) on trial functions [keeping the relation (6.2) true] in addition to the symmetry requirements, the solution $E^{\Omega}$ approximates only this excited energy. As follows from Sec. VI A, all results obtained in Secs. II-V must hold also for these excited (exact or approximate) states.

It should be noted that in terms of the above-mentioned symmetry-restricted variational problem, the GS energy is also the lowest energy of some particular symmetry, but, in addition, it represents the absolute minimum when all possible symmetries are considered. It may happen for some systems, however, that the symmetry of the GS may be different in various regions of the $(R, B)$ plane. In such a case the results (4.22), (4.28), and (4.33) and also Eqs. (4.35)(4.37), should be used with caution. As obtained by integration of partial differential equations, they are correct under assumption that $T_{0 \overline{2}}, E_{\mathrm{C}}$, and $E_{\mathrm{fd}}$, respectively, are continuous functions of $R^{\prime}, B^{\prime}$. But these functions may show discontinuity at the border between regions of different symmetries for the GS solution. To satisfy the mentioned assumption, one should take these functions selected by the criterion of the symmetry of the solution (in the whole range of integration over $R^{\prime}, B^{\prime}$ ) rather than by the GS criterion.

Inspired by the very recent Görling paper [17], we are going to argue that our results for the field dependence hold also for arbitrary excited-state exact energies. A convenient tool (invoked by Görling) — the basic stationarity principle of quantum mechanics - will play for excited states the same role that the variational principle (2.22) played for the GS. According to this principle, the eigensolution $\left\{E^{\kappa}[\mathbf{V}, \mathbf{A}], \Psi^{\kappa}[\mathbf{V}, \mathbf{A}]\right\}$ of the Schrödinger equation

$$
\hat{H}[\mathrm{v}, \mathbf{A}] \Psi^{\kappa}[\mathrm{v}, \mathbf{A}]=E^{\kappa}[\mathrm{v}, \mathbf{A}] \Psi^{\kappa}[\mathrm{v}, \mathbf{A}]
$$

leads to the stationary expectation value of the Hamiltonian

$$
\begin{gathered}
\langle\Psi(\varepsilon)|\hat{H}| \Psi(\varepsilon)\rangle=E^{\kappa}+O\left(\varepsilon^{2}\right) \\
\text { for } \Psi(\varepsilon) \in \Omega, \quad \Psi(\varepsilon)=\Psi^{\kappa}+\varepsilon \delta \Psi+O\left(\varepsilon^{2}\right)
\end{gathered}
$$

(vanishing first variation). The set $\Omega$ is the same as that defined at the beginning of Sec. VI B. Thus the composite label $\kappa$ includes the label of the irreducible representation of $\Omega$ and the "principal" quantum number which orders the eigenvalues of the given symmetry into an increasing sequence. It should be noted that the symmetry group of the Hamiltonian of a molecule in homogeneous MF remains the same for all $\{R, B\}$ from the region $0<R<\infty, 0<B<\infty$.

Similarly as for the GS case, we define the scaled function $\Psi_{\xi}^{\kappa}$ by applying Eq. (2.21) to $\Psi^{\kappa}$. According to Eq. (6.7), the analog of Eq. (2.23) is true

$$
0=\left.\frac{\partial}{\partial \xi}\left\langle\Psi_{\xi}^{\kappa}|\hat{H}[\mathbf{v}, \mathbf{A}]| \Psi_{\xi}^{\kappa}\right\rangle\right|_{\xi=1},
$$

so all remaining equations of Sec. II B hold for the state $\kappa$. In order to have the Hellmann-Feynman theorem

$$
\frac{d}{d \epsilon} E^{\kappa}(\epsilon)=\left\langle\Psi^{\kappa}(\epsilon)\left|\frac{d \hat{H}(\epsilon)}{d \epsilon}\right| \Psi^{\kappa}(\epsilon)\right\rangle
$$

(and, therefore, the results of Sec. II C) extended to the excited states, we need the eigensolution $\left\{E^{\kappa}\left(\epsilon^{\prime}\right), \Psi^{\kappa}\left(\epsilon^{\prime}\right)\right\}$ of the Hamiltonian $\hat{H}\left(\epsilon^{\prime}\right)$ to be labeled by the same $\kappa$ as the eigensolution of the close Hamiltonian $\hat{H}(\epsilon)$, i.e., for $\mid \epsilon^{\prime}$ $-\epsilon \mid \ll 1$, the eigenenergy $E^{\kappa}\left(\epsilon^{\prime}\right)$ should be close to $E^{\kappa}(\epsilon)$, but far from $E^{\kappa^{\prime}}(\epsilon)$ for $\kappa^{\prime} \neq \kappa$ (the separability property). Then the proof of Eq. (6.9) goes similarly as in Eq. (6.4), but now $d\left\langle\Psi^{\kappa}\left(\epsilon^{\prime}\right)|\hat{H}(\epsilon)| \Psi^{\kappa}\left(\epsilon^{\prime}\right)\right\rangle / d \epsilon^{\prime}=0$ at $\epsilon^{\prime}=\epsilon$ takes place due to the stationarity principle (6.7).

Thus all results concerning the VEs, obtained for the GS, hold also for excited states. However, in connection with the separability, these results are not available for such states $\kappa$ at a particular point $\{R, B\}$, for which an accidental degeneracy occurs, $E^{\kappa}(R, B)=E^{\kappa^{\prime}}(R, B)$.

\section{Magnetic Thomas-Fermi solutions}

The statistical model of an atom in the absence of a magnetic field, known as the Thomas-Fermi approximation (see, e.g., in Refs. $[9,14]$ ), has been extended to an intense magnetic field by Kadomtsev [10] (see also Mueller, Rau, and Spruch [11]). It will be called here the magnetic ThomasFermi (MTF) approximation. For a molecule characterized by the electron number $N$ and the external potential $\mathrm{V}_{\mathrm{en}}(\mathbf{r} ; \mathbf{Z}, \mathbf{R})$ of its nuclear skeleton, Eq. (3.1), immersed in a homogeneous MF $B$, the GS energy $E^{\mathrm{MTF}}(N, \mathbf{Z}, \mathbf{R}, B)$ and the electron-number density $n^{\mathrm{MTF}}(\mathbf{r} ; N, \mathbf{Z}, \mathbf{R}, B)$ in the MTF approximation represent the solution of the variational problem

$$
\begin{aligned}
& E^{\mathrm{MTF}}(N, \mathbf{Z}, \mathbf{R}, B)=\min _{n \rightarrow N} \widetilde{E}^{\mathrm{MTF}}[n, \mathbf{Z}, \mathbf{R}, B] \\
& =\widetilde{E}^{\mathrm{MTF}}\left[n^{\mathrm{MTF}}(. ; N, \mathbf{Z}, \mathbf{R}, B), \mathbf{Z}, \mathbf{R}, B\right],
\end{aligned}
$$

where the variational density $n(\mathbf{r}) \geqslant 0$ is constrained by the requirement

$$
\int d^{3} r n(\mathbf{r})=N
$$

The MTF energy functional is a sum

$$
\widetilde{E}^{\mathrm{MTF}}[n, \mathbf{Z}, \mathbf{R}, B]=\widetilde{E}_{\mathrm{kin}}[n, B]+\widetilde{E}_{\mathrm{en}}[n, \mathbf{Z}, \mathbf{R}]+\widetilde{E}_{\mathrm{es}}[n]
$$

of the kinetic energy functional

$$
\widetilde{E}_{\mathrm{kin}}[n, B]=c_{\mathrm{MTF}} B^{-2} \int d^{3} r n^{3}(\mathbf{r}),
$$

where $c_{\mathrm{MTF}}=2 \pi^{4} \hbar^{4} /\left(3 m e^{2}\right)$, which replaces the sum $\left(\widetilde{T}_{0}\right.$ $+\widetilde{T}_{1}+\widetilde{T}_{2}+\widetilde{T}_{1}$ ) of the exact theory terms, Eq. (2.4); the electron-nuclei energy functional

$$
\widetilde{E}_{\mathrm{en}}[n, \mathbf{Z}, \mathbf{R}]=\int d^{3} r n(\mathbf{r}) \mathrm{v}_{\mathrm{en}}(\mathbf{r} ; \mathbf{Z}, \mathbf{R}),
$$

which is the same as in the exact theory; and the classical electrostatic energy of electrons 


$$
\widetilde{E}_{\mathrm{es}}[n]=\frac{e^{2}}{4 \pi \varepsilon_{0}} \int d^{3} r_{1} d^{3} r_{2} \frac{n\left(\mathbf{r}_{1}\right) n\left(\mathbf{r}_{2}\right)}{2\left|\mathbf{r}_{1}-\mathbf{r}_{2}\right|}
$$

which approximates the $\widetilde{E}_{\mathrm{ee}}$ term of the exact theory, Eq. (2.10) with (2.3). The solution of Eq. (6.10a) is known to exist [18] for $N \leqslant Z$, where $Z=\Sigma_{l} Z_{l}$. The MTF model is valid $[10,11]$ for MFs $B$ satisfying

$$
Z^{4 / 3} \ll B / B_{\text {au }} \ll Z^{3},
$$

where $B_{\mathrm{au}}=\hbar /\left(e a_{\mathrm{B}}^{2}\right)=2.3506 \times 10^{5} \mathrm{~T}$ is the atomic unit of the field $\left(1 \mathrm{~T}=10^{4} \mathrm{G}\right)$.

In analogy with Eq. (3.9a) we define the total energy of a molecule in the MTF approximation as

$$
\begin{aligned}
E_{\mathrm{mol}}^{\mathrm{MTF}}(N, \mathbf{Z}, \mathbf{R}, B) & =E^{\mathrm{MTF}}(N, \mathbf{Z}, \mathbf{R}, B)+E_{\mathrm{nn}}(\mathbf{Z}, \mathbf{R}) \\
& =E_{\mathrm{kin}}(N, \mathbf{Z}, \mathbf{R}, B)+E_{\mathrm{C}}^{\mathrm{MTF}}(N, \mathbf{Z}, \mathbf{R}, B),
\end{aligned}
$$

where $E_{\mathrm{nn}}$ is given in Eq. (3.8) and the sum of all Coulombic terms is approximated now by

$$
E_{\mathrm{C}} \rightarrow E_{\mathrm{C}}^{\mathrm{MTF}}=E_{\mathrm{en}}+E_{\mathrm{es}}+E_{\mathrm{nn}}
$$

[compare Eq. (3.10b)]. Here $E_{\text {kin }}(N, \mathbf{Z}, \mathbf{R}, B)$ $=\widetilde{E}_{\text {kin }}\left[n^{\mathrm{MTF}}(. ; N, \mathbf{Z}, \mathbf{R}, B), B\right]$, and similarly for other terms. As in Sec. IV B, we are going to find the virial relations for a uniformly scaled molecule, Eq. (4.10), which is characterized by the size parameter $R$. Now we simplify the notation $E_{\mathrm{mol}}^{\mathrm{MTF}}\left(N, \mathbf{Z}, R \mathbf{R}^{0}, B\right) \rightarrow E_{\mathrm{mol}}^{\mathrm{MTF}}(N, R, B)$, and similarly for all components of the total energy and for the density, by suppressing the dependence on the fixed $\mathbf{Z}$ and $\mathbf{R}^{0}$.

By applying the variational Hellmann-Feynman identity (6.5) to the variational MTF solution (6.10a) (here in terms of the density rather than the wave function) we find

$$
\frac{\partial}{\partial B} E^{\mathrm{MTF}}(N, R, B)=\left.\frac{\partial}{\partial B^{\prime}} \widetilde{E}_{\mathrm{kin}}\left[n^{\mathrm{MTF}}(. ; N, R, B), B^{\prime}\right]\right|_{B^{\prime}=B},
$$

$$
\frac{\partial}{\partial R} E^{\mathrm{MTF}}(N, R, B)=\left.\frac{\partial}{\partial R^{\prime}} \widetilde{E}_{\mathrm{en}}\left[n^{\mathrm{MTF}}(. ; N, R, B), R^{\prime}\right]\right|_{R^{\prime}=R} .
$$

After performing differentiation of $\widetilde{E}_{\text {kin }}$ given in the form (6.10d), and taking into account that $E_{\mathrm{nn}}$ is independent of $B$, we obtain for the field dependence of the total molecular energy (6.11) the following result:

$$
B \frac{\partial}{\partial B} E_{\mathrm{mol}}^{\mathrm{MTF}}(N, R, B)=-2 E_{\mathrm{kin}}(N, R, B) .
$$

On the way to obtain the VE we define the following scaled density:

$$
n_{\xi}(\mathbf{r})=\xi^{3} n^{\mathrm{MTF}}(\xi \mathbf{r} ; N, R, B),
$$

Since this density satisfies the constraint (6.10b) and for $\xi$ $=1$ coincides with the solution of Eq. (6.10a), it can serve as a trial density for an alternative determination of the MTF GS energy (6.10a) by the following minimization:

$$
E^{\mathrm{MTF}}(N, R, B)=\min _{\xi} \widetilde{E}^{\mathrm{MTF}}\left[n_{\xi}, R, B\right]=\widetilde{E}^{\mathrm{MTF}}\left[n_{\xi=1}, R, B\right] .
$$

Therefore, the necessary condition

$$
0=\left.\frac{\partial}{\partial \xi} \widetilde{E}^{\mathrm{MTF}}\left[n_{\xi}, R, B\right]\right|_{\xi=1}
$$

must hold. Using Eq. (6.15) and the definitions (6.10d)(6.10f), we find the explicit $\xi$ dependence of various terms in Eq. (6.16) with (6.10c):

$$
\begin{aligned}
& \widetilde{E}_{\mathrm{kin}}\left[n_{\xi}, B\right]=c_{\mathrm{MTF}} B^{-2} \xi^{6} \int d^{3} r^{\prime}\left[n^{\mathrm{MTF}}\left(\mathbf{r}^{\prime} ; R, B\right)\right]^{3} \\
&=\xi^{6} \widetilde{E}_{\mathrm{kin}}\left[n^{\mathrm{MTF}}(. ; R, B), B\right] \\
& \widetilde{E}_{\mathrm{en}}\left[n_{\xi}, R\right]= \xi \int d^{3} r^{\prime} n^{\mathrm{MTF}}\left(\mathbf{r}^{\prime} ; R, B\right) \mathrm{v}_{\mathrm{en}}\left(\mathbf{r}^{\prime} ; \mathbf{Z}, \xi R \mathbf{R}^{0}\right) \\
&= \xi \widetilde{E}_{\mathrm{en}}\left[n^{\mathrm{MTF}}(. ; R, B), \xi R\right], \\
& \widetilde{E}_{\mathrm{es}}\left[n_{\xi}\right]=\xi \widetilde{E}_{\mathrm{es}}\left[n^{\mathrm{MTF}}(. ; R, B)\right] .
\end{aligned}
$$

So the differentiation in Eq. (6.17) leads to

$$
0=6 E_{\mathrm{kin}}+E_{\mathrm{en}}+E_{\mathrm{es}}+\left.\frac{\partial}{\partial \xi} \widetilde{E}_{\mathrm{en}}\left[n^{\mathrm{MTF}}(. ; R, B), \xi R\right]\right|_{\xi=1} .
$$

Finally, using Eqs. (6.14), (6.13b), and (6.10c), the VE is obtained in the form

$$
0=3 E_{\text {kin }}(N, R, B)+\left(1+R \frac{\partial}{\partial R}-B \frac{\partial}{\partial B}\right) E^{\mathrm{MTF}}(N, R, B),
$$

or, using Eqs. (6.11) and (3.11), in the form

$$
\left(-R \frac{\partial}{\partial R}+B \frac{\partial}{\partial B}-1\right) E_{\mathrm{mol}}^{\mathrm{MTF}}(N, R, B)=3 E_{\mathrm{kin}}(N, R, B) .
$$

We see that this VE for the MTF approximation is the same in form as for the exact GS solution if the replacement

$$
T_{02} \rightarrow 3 E_{\text {kin }}
$$

is performed in Eq. (4.12).

By subtracting Eq. (6.11) from Eq. (6.14) we have

$$
\begin{aligned}
\left(B \frac{\partial}{\partial B}-1\right) E_{\mathrm{mol}}^{\mathrm{MTF}}(N, R, B)= & -3 E_{\mathrm{kin}}(N, R, B) \\
& -E_{\mathrm{C}}^{\mathrm{MTF}}(N, R, B) .
\end{aligned}
$$

Thus the combination of Eq. (6.23) and (6.25) leads to an alternative form of the VE 
$\left(-\frac{1}{2} R \frac{\partial}{\partial R}+B \frac{\partial}{\partial B}-1\right) E_{\mathrm{mol}}^{\mathrm{MTF}}(N, R, B)=-\frac{1}{2} E_{\mathrm{C}}^{\mathrm{MTF}}(N, R, B)$,

analogous to the VE (4.13) for the exact solution, and to the size dependence

$$
\begin{aligned}
R \frac{\partial}{\partial R} E_{\mathrm{mol}}^{\mathrm{MTF}}(N, R, B)= & -\left\{E_{\mathrm{C}}^{\mathrm{MTF}}(N, R, B)\right. \\
& \left.+2\left[3 E_{\mathrm{kin}}(N, R, B)\right]\right\},
\end{aligned}
$$

analogous to Eq. (4.14) with replacements (6.12) and (6.24).

It is interesting that in the MTF approximation the derivatives of $E^{\mathrm{MTF}}$ can be determined not only with respect to $B$ and $R$, Eqs. (6.14) and (6.27), but also with respect to $N$. This is possible due to the fact that the dependence on $N$ enters into Eq. (6.10a) only via the constraint $(6.10 b)$ and that it remains meaningful for any real $N>0$, not only for integers. With the help of a Lagrange multiplier $\mu^{\text {MTF }}$ the minimization (6.10a), constrained by Eq. (6.10b), leads to

$$
\mu^{\mathrm{MTF}}=\mathrm{const}=\left.\frac{\delta \widetilde{E}^{\mathrm{MTF}}[n, R, B]}{\delta n(\mathbf{r})}\right|_{n=n^{\mathrm{MTF}}} .
$$

Next, differentiating Eq. (6.10a) and using the result (6.28) we find

$$
\begin{aligned}
\frac{\partial}{\partial N} E^{\mathrm{MTF}}(N, R, B)= & \left.\int d^{3} r \frac{\delta \widetilde{E}^{\mathrm{MTF}}[n, R, B]}{\delta n(\mathbf{r})}\right|_{n=n^{\mathrm{MTF}}} \\
& \times \frac{\partial n^{\mathrm{MTF}}(\mathbf{r} ; N, R, B)}{\partial N} \\
= & \mu^{\mathrm{MTF}} \frac{\partial}{\partial N} \int d^{3} r n^{\mathrm{MTF}}(\mathbf{r} ; N, R, B)=\mu^{\mathrm{MTF}}
\end{aligned}
$$

Knowing the explicit form of the functional dependence of $\widetilde{E}^{\mathrm{MTF}}$ on $n(\mathbf{r})$, Eqs. (6.10c)-(6.10f), we can find easily the functional derivative $\delta \widetilde{E}^{\mathrm{MTF}} / \delta n(\mathbf{r})$. Now, using this result, we multiply both sides of Eq. (6.28) by $n^{\mathrm{MTF}}(\mathbf{r} ; N, R, B)$, integrate over $\mathbf{r}$, and substitute for $\mu^{\mathrm{MTF}}$ the result (6.29), to obtain

$$
N \frac{\partial}{\partial N} E^{\mathrm{MTF}}(N, R, B)=3 E_{\mathrm{kin}}+E_{\mathrm{en}}+2 E_{\mathrm{es}} .
$$

Since the MTF approximation is valid for a sufficiently strong field only, Eq. (6.10g), we cannot obtain analogs of the results from Secs. IV C-IV E, because the $B \rightarrow 0$ limit is involved there. However, the results obtained in Sec. IV F are valid for the MTF approximation, provided $B_{0}$ is in the range $(6.10 \mathrm{~g})$ and the replacements $(6.12)$ and (6.24) are applied in Eq. (4.35)-(4.37).

Similarly as for molecules, all results of Sec. V for atoms [except Eqs. (5.9) and (5.10)] are valid for the MTF approximation when the replacements (6.24) and the atomic analog of Eq. (6.12), $E_{\mathrm{C}} \rightarrow E_{\mathrm{C}}^{\mathrm{MTF}}=E_{\mathrm{en}}+E_{\mathrm{es}}$, are applied. In particular, for the analog of Eq. (5.7) we have

$$
E_{\mathrm{en}}(N, Z, B)+E_{\mathrm{es}}(N, Z, B)+6 E_{\mathrm{kin}}(N, Z, B)=0,
$$

the VE indicated already by Mueller et al. [11]. In Appendix $\mathrm{B}$ we show that this $\mathrm{VE}$ and also the extrapolating expression (5.11) are satisfied by the known solution of Eq. (6.10) for an atom.

\section{SUMMARY}

In conclusion, the main results are summed up in the following.

(i) The virial equation for a many-electron system in the fields of arbitrary scalar $\mathrm{v}(\mathbf{r})$ and vector $\mathbf{A}(\mathbf{r})$ potentials is obtained in three equivalent forms (2.30), (2.31), and (2.37). The last one represents a generalization of the result of Erhard and Gross [16] by including the B-dependent term.

(ii) For a molecule (neutral or ionized) in the field of an arbitrary vector potential $\mathbf{A}(\mathbf{r})$ the obtained virial equation (3.12) [with Eq. (2.36)] generalizes the well known VE for a molecule in the absence of a MF.

(iii) For a molecule in a homogeneous $\mathrm{MF}, \mathbf{B}=$ const, the result (4.5) gives the field derivative of the molecular energy, while Eqs. (4.6) and (4.8) provide three forms of the VE. After introducing a uniformly scaled (squeezed/stretched) molecule, characterized by the size parameter $R$, an expression for the derivative of the molecular energy $E_{\text {mol }}(R, B)$ with respect to $R$ is obtained in Eq. (4.14), while the two virial equations represent partial differential equations (4.12) and (4.13a) for this energy, having the solutions (4.22) and (4.28): the molecular energy for the given size $R$ and the MF $B$ is obtained in terms of the molecular energy and the magnetization vectors (of separate atoms), both in the absence of a MF, and a specific integral involving either the kinetic term $\left(T_{0}-T_{2}\right)$, or the total Coulombic contribution $E_{\mathrm{C}}$, integrated over sizes larger than $R$ and fields smaller than $B$. It is worth noting that the $T_{1}$ term, involving the paramagneticcurrent density vector (which is characteristic for the densityfunctional theory generalized to the MF case, see, e.g. Refs. $[16,15]$ ), and the $T_{1}$, term, involving the magnetization density, both are absent in these solutions. When the geometry of a molecule for the given $R$ is kept fixed, the fielddepending energy is given by Eq. (4.33): it involves the molecular energy and magnetization vector, both in the absence of a MF, and the sum $\left(T_{0}-T_{2}+E_{\mathrm{C}}\right)$, a function of $R$ and $B^{\prime}$, integrated over $B^{\prime}$ smaller than $B$. Eqs. (4.35)-(4.37) provide an extrapolation of results known at the field $B_{0}$ to the field $B$.

(iv) Analogous results for an atom in a homogeneous $\mathrm{MF}$ are given in Eqs. (5.3)-(5.11).

It is interesting to see how the well known virial relation for an atom in the absence of a MF, Eq. (5.8), is modified to include $B$, Eq. (5.7), and next, when generalized for a molecule, it remains in the same form at the equilibrium size, Eq. (4.16), while at an arbitrary size it holds in an "averaged' sense, Eq. (4.29). It should be stressed that all results for the field-dependence concern not only the exact GS energy, but also exact excited-state energies, and a wide class of approximate (GS and some excited-state) energies, resulting from variational solutions, Eq. (6.1), among them, the Hartree-Fock approximation. Even the results for the field 
dependence of the magnetic Thomas-Fermi energy, Eq. (6.10), show the same form, provided the substitutions (6.12) and (6.24) are applied.

\section{ACKNOWLEDGMENTS}

One of us (N.H.M.) acknowledges partial financial support for this work from the ONR. Dr. P. Schmidt of that office is to be thanked for his continuing interest and constant encouragement. Professor Yu Lu and his colleagues are to be thanked by both of us for a stimulating environment at ICTP, Trieste, in which much of the present work was carried out, as well as for their hospitality in Trieste.

\section{APPENDIX A: GAUGE INVARIANCE} by

The transformation of the vector potential $\mathbf{A}(\mathbf{r})$, defined

$$
\mathbf{A}(\mathbf{r}) \rightarrow \mathbf{A}^{\text {new }}(\mathbf{r})=\mathbf{A}(\mathbf{r})-\boldsymbol{\nabla} \Lambda(\mathbf{r}),
$$

where $\Lambda(\mathbf{r})$ is an arbitrary scalar function, does not change a physical quantity — the magnetic field $\mathbf{B}(\mathbf{r})$, Eq. (2.1). It is known that the eigenfunction $\Psi^{\text {new }}$ of the Schrödinger equation (2.19) in which $\hat{H}$ is replaced by $\hat{H}^{\text {new }}$;

$$
\hat{H}[\mathrm{v}, \mathbf{A}] \rightarrow \hat{H}^{\text {new }} \equiv \hat{H}\left[\mathrm{v}, \mathbf{A}^{\text {new }}\right],
$$

differs from the eigenfunction $\Psi=\Psi[\mathrm{V}, \mathbf{A}]$ of the original Schrödinger equation by a $\Lambda$-dependent phase factor only

$$
\begin{aligned}
\Psi \rightarrow & \Psi^{\text {new }}\left(\mathbf{x}_{1}, \ldots, \mathbf{x}_{N}\right) \\
& =\exp \left(i \frac{e}{\hbar}\left[\Lambda\left(\mathbf{r}_{1}\right)+\ldots+\Lambda\left(\mathbf{r}_{N}\right)\right]\right) \Psi\left(\mathbf{x}_{1}, \ldots, \mathbf{x}_{N}\right),
\end{aligned}
$$

while the eigenenergy is independent of the unphysical gauge function $\Lambda(\mathbf{r})$

$$
E^{\text {new }} \equiv\left\langle\Psi^{\text {new }}\left|\hat{H}^{\text {new }}\right| \Psi^{\text {new }}\right\rangle=\langle\Psi|\hat{H}| \Psi\rangle \equiv E .
$$

The transformed DMs are defined by Eqs. (2.14)-(2.18) applied to the transformed wave function $\Psi^{\text {new }}$. One sees immediately that the diagonal (in spatial indices) DMs are gauge invariant, $\quad n^{\text {new }}=n, \quad n_{2}^{\text {new }}=n_{2}, \quad \mathbf{m}^{\text {new }}=\mathbf{m}\left[\gamma_{1}^{\text {new }}\right]$ $=\mathbf{m}\left[\gamma_{1}\right]$, Eq. (2.13). Therefore, from Eqs. (2.8)-(2.10) we find

$$
T_{1^{\prime}}^{\text {new }}=T_{1^{\prime}}, \quad V^{\text {new }}=V, \quad E_{\mathrm{ee}}^{\text {new }}=E_{\mathrm{ee}} .
$$

The transformation of the 1DM, induced by Eq. (A3),

$$
\rho_{1}^{\text {new }}\left(\mathbf{r}_{1} ; \mathbf{r}_{1}^{\prime}\right)=\exp \left(i \frac{e}{\hbar}\left[\Lambda\left(\mathbf{r}_{1}\right)-\Lambda\left(\mathbf{r}_{1}^{\prime}\right)\right]\right) \rho_{1}\left(\mathbf{r}_{1} ; \mathbf{r}_{1}^{\prime}\right)
$$

leads, according to Eqs. (2.11) and (2.12), to

$$
\begin{aligned}
t^{\text {new }}(\mathbf{r}) \equiv & t\left(\mathbf{r} ;\left[\rho_{1}^{\text {new }}\right]\right)=t(\mathbf{r})+e \mathbf{j}_{p}(\mathbf{r}) \cdot \boldsymbol{\nabla} \Lambda(\mathbf{r})+\frac{e^{2}}{2 m} n(\mathbf{r}) \\
& \times[\boldsymbol{\nabla} \Lambda(\mathbf{r})]^{2}
\end{aligned}
$$

$$
\mathbf{j}_{\mathrm{p}}^{\text {new }}(\mathbf{r}) \equiv \mathbf{j}_{\mathrm{p}}\left(\mathbf{r} ;\left[\rho_{1}^{\text {new }}\right]\right)=\mathbf{j}_{\mathrm{p}}(\mathbf{r})+\frac{e}{m} n(\mathbf{r}) \boldsymbol{\nabla} \Lambda(\mathbf{r}) .
$$

Using these results in Eqs. (2.5)-(2.7), one verifies immediately by simple algebra that

$$
\begin{aligned}
T_{0}^{\text {new }}+T_{1}^{\text {new }}+T_{2}^{\text {new }} \equiv & \int d^{3} r\left\{t^{\text {new }}(\mathbf{r})+e \mathbf{j}_{\mathrm{p}}^{\text {new }}(\mathbf{r}) \cdot[\mathbf{A}(\mathbf{r})\right. \\
& \left.-\boldsymbol{\nabla} \Lambda(\mathbf{r})]+\frac{e^{2}}{2 m} n(\mathbf{r})[\mathbf{A}(\mathbf{r})-\boldsymbol{\nabla} \Lambda(\mathbf{r})]^{2}\right\} \\
= & \int d^{3} r\left\{t(\mathbf{r})+e \mathbf{j}_{\mathrm{p}}(\mathbf{r}) \cdot \mathbf{A}(\mathbf{r})\right. \\
& \left.+\frac{e^{2}}{2 m} n(\mathbf{r}) \mathbf{A}^{2}(\mathbf{r})\right\} \equiv T_{0}+T_{1}+T_{2} . \quad(\text { A9 })
\end{aligned}
$$

Thus combining Eqs. (A5) and (A9) we check that the total energy, Eq. (2.20), is gauge invariant, $E^{\text {new }}=E$, as announced in Eq. (A4).

Let us find now the gauge transformation of the kinetic energy combination entering the VE

$$
\begin{aligned}
T_{0}^{\text {new }} & -T_{2}^{\text {new }} \\
& \equiv \int d^{3} r\left\{t^{\text {new }}(\mathbf{r})-\frac{e^{2}}{2 m} n(\mathbf{r})[\mathbf{A}(\mathbf{r})-\boldsymbol{\nabla} \Lambda(\mathbf{r})]^{2}\right\} \\
& =T_{0}-T_{2}+\int d^{3} r e\left\{\mathbf{j}_{\mathrm{p}}(\mathbf{r})+\frac{e}{m} n(\mathbf{r}) \mathbf{A}(\mathbf{r})\right\} \cdot \boldsymbol{\nabla} \Lambda(\mathbf{r}) \\
& =T_{0}-T_{2}-\int d^{3} r \Lambda(\mathbf{r}) e \boldsymbol{\nabla} \cdot\left\{\mathbf{j}_{\mathrm{p}}(\mathbf{r})+\frac{e}{m} n(\mathbf{r}) \mathbf{A}(\mathbf{r})\right\} .
\end{aligned}
$$

(Vanishing of the DMs and their derivatives at infinite distances has been used in the last step.) The vector represented by the combination in the curly braces - the physical current $\mathbf{j}(\mathbf{r})$ - is known to satisfy the continuity equation (for stationary states)

$$
\boldsymbol{\nabla} \cdot \mathbf{j}(\mathbf{r}) \equiv \boldsymbol{\nabla} \cdot\left\{\mathbf{j}_{\mathrm{p}}(\mathbf{r})+\frac{e}{m} n(\mathbf{r}) \mathbf{A}(\mathbf{r})\right\}=0 .
$$

Thus the last integral in Eq. (A10) vanishes, so $\left(T_{0}-T_{2}\right)$ is gauge invariant.

\section{APPENDIX B: EXAMPLE OF THE MTF ATOM}

As shown by Banerjee, Constantinescu, and Rehák [12], the GS energy of an atom in the MTF approximation, the solution of Eq. (6.10), can be written as

$$
E^{\mathrm{MTF}}(N, Z, B)=Z^{9 / 5} B^{2 / 5} \epsilon(N / Z),
$$

where $\epsilon(\nu)$ is a universal function defined for $0<\nu \leqslant 1$, available numerically [the authors provide results for $\nu$ $=1,(1-1 / Z),(1-2 / Z)$ at $Z=5,6, \ldots, 100]$. For small $\nu$ this function was shown by March and Tomishima [13] to have an expansion

$$
\epsilon(\nu)=\text { const } \nu^{3 / 5}[1+O(\nu)] .
$$


We are going to verify now that this solution (B1) satisfies the VE (6.31) and the extrapolating expression (5.11). It will be convenient to introduce an auxiliary function

$$
\varphi(\nu)=\frac{d \ln (\epsilon(\nu))}{d \ln (\nu)}, \quad \text { so } \varphi(\nu)=\frac{3}{5}+O(\nu) \text { for } \nu \ll 1,
$$

in terms of which the derivatives of $E^{\mathrm{MTF}}$, Eq. (B1), can be written

$$
\begin{gathered}
N \frac{\partial}{\partial N} E^{\mathrm{MTF}}(N, Z, B)=\varphi(N / Z) E^{\mathrm{MTF}}(N, Z, B), \\
Z \frac{\partial}{\partial Z} E^{\mathrm{MTF}}(N, Z, B)=[(9 / 5)-\varphi(N / Z)] E^{\mathrm{MTF}}(N, Z, B), \\
B \frac{\partial}{\partial B} E^{\mathrm{MTF}}(N, Z, B)=(2 / 5) E^{\mathrm{MTF}}(N, Z, B) .
\end{gathered}
$$

But the same derivatives are known to be linear combinations of $E_{\text {kin }}, E_{\text {en }}$, and $E_{\text {es }}$, Eqs. (6.30), (5.3), and (6.14). The solution of such a system of 3 linear equations can be found immediately to be

$$
\begin{gathered}
E_{\mathrm{kin}}=-\frac{1}{5} E^{\mathrm{MTF}}, \\
E_{\mathrm{en}}=\left(\frac{9}{5}-\varphi\right) E^{\mathrm{MTF}}, \\
E_{\mathrm{es}}=\left(\varphi-\frac{3}{5}\right) E^{\mathrm{MTF}} .
\end{gathered}
$$

After substituting these values into Eq. (6.31) we see that this VE is satisfied identically. No explicit form of the function $\epsilon(\nu)$ was involved, therefore the VE (6.31) is not helpful in checking the numerical accuracy of the solution (B1).

In order to verify that the solution (B1) satisfies the extrapolating expression (5.11) for $E(B) \rightarrow E^{\mathrm{MTF}}(N, Z, B)$, we need to perform the replacement (6.24) using Eq. (B7):

$$
\begin{aligned}
E^{\mathrm{MTF}}(N, Z, B) & =\left(B / B_{0}\right) E^{\mathrm{MTF}}\left(N, Z, B_{0}\right)+\int_{B_{0} / B}^{1} d \zeta \zeta^{-2}(-3 / 5) E^{\mathrm{MTF}}(N, Z, B \zeta) \\
& =Z^{9 / 5} \epsilon(N / Z)\left\{B B_{0}^{-3 / 5}+B^{2 / 5} \int_{B_{0} / B}^{1} d \zeta(-3 / 5) \zeta^{-8 / 5}\right\} \\
& =Z^{9 / 5} \epsilon(N / Z)\left\{B B_{0}^{-3 / 5}+B^{2 / 5}\left(1-\left(B_{0} / B\right)^{-3 / 5}\right)\right\} \\
& =Z^{9 / 5} \epsilon(N / Z) B^{2 / 5} .
\end{aligned}
$$

So, really, the solution (B1) is recovered by means of the extrapolation.

[1] Atoms and Molecules in Intense Fields, edited by L. S. Cederbaum, K. C. Kulander, and N. H. March, Vol. 86 of Structure and Bonding Series (Springer-Verlag, Berlin, 1997).

[2] H. Ruder, G. Wunner, H. Herold, and F. Geyer, Atoms in Strong Magnetic Fields (Springer-Verlag, Berlin, 1994).

[3] Atoms in Strong Fields, edited by M. Gavrila (Academic, Boston, 1992).

[4] G. Chanmugam, Annu. Rev. Astron. Astrophys. 30, 143 (1992).

[5] G. R. Freeman and N. H. March, J. Phys. Chem. 100, 4331 (1996).

[6] M. K. Scheller, R. N. Compton, and L. S. Cederbaum, Science 270, 1160 (1995).

[7] M. D. Jones, G. Ortiz, and D. M. Ceperley, Phys. Rev. A 54, 219 (1996)

[8] M. Demeur, P.-H. Heenen, and M. Godefroid, Phys. Rev. A 49, 176 (1994).
[9] N. H. March, Electron Density Theory of Atoms and Molecules (Academic, London, 1992).

[10] B. B. Kadomtsev, Zh. Éksp. Teor. Fiz. 58, 1765 (1970) [Sov. Phys. JETP 31, 945 (1970)].

[11] R. O. Mueller, A. R. P. Rau, and L. Spruch, Phys. Rev. Lett. 26, 1136 (1971)

[12] B. Banerjee, D. H. Constantinescu, and P. Rehák, Phys. Rev. D 10, 2384 (1974).

[13] N. H. March and Y. Tomishima, Phys. Rev. D 19, 449 (1979).

[14] R. G. Parr and W. Yang, Density-Functional Theory of Atoms and Molecules (Oxford University Press, Oxford, 1989).

[15] A. Holas and N. H. March, Phys. Rev. A 56, 4595 (1997).

[16] S. Erhard and E. K. U. Gross, Phys. Rev. A 53, R5 (1996).

[17] A. Görling, Phys. Rev. A 59, 3359 (1999).

[18] E. H. Lieb, J. P. Solovej, and J. Yngvason, Commun. Math. Phys. 161, 77 (1994). 